\title{
Object identification without foveal vision: Evidence from an artificial scotoma paradigm
}

\author{
JOHN M. HENDERSON, KAREN K. MCCLURE, STEVEN PIERCE, and GARY SCHROCK \\ Michigan State University, East Lansing, Michigan
}

\begin{abstract}
The purpose of the present study was to investigate the degree to which foveal information is necessary for object identification. To explore this question, we used an artificial moving scotoma paradigm to eliminate a region of a currently viewed display in real time as a function of eye position. Subjects examined linear arrays of four line drawings of objects while their eye movements were recorded. Immediately following each array, a test probe was presented to test the degree to which the subject had identified those objects. Three conditions were compared, one in which a central foveal scotoma was created, one in which an extrafoveal scotoma was created, and a control condition in which the scotoma was absent. The main result was that identification accuracy was very good, but eye-movement behavior was disrupted when a foveal scotoma was present. These results suggest that foveal information is not necessary but is beneficial for perceptual encoding during object identification; the loss of foveal information can be overcome as long as compensatory extrafoveal processing is possible.
\end{abstract}

What is the purpose of saccadic eye movements in object encoding? Most vision scientists would agree that saccades are used to bring an area of interest in a scene to the high-acuity fovea. Given that the majority of fixations during scene perception are centered on or very close to objects rather than on empty space or background (Buswell, 1935; Yarbus, 1967), foveal fixation seems to provide for high-acuity object analysis. What is less clear, however, is the degree to which the acquisition of detailed foveal information is necessary for object identification.

\section{Transsaccadic Integration Studies}

One source of evidence that useful information can be acquired from objects beyond the fovea is provided by studies examining object identification across saccades. These studies have been modeled after similar experiments in word identification (Henderson, Dixon, Petersen, Twilley, \& Ferreira, 1995; Rayner, 1975; Rayner, McConkie, \& Ehrlich, 1978), and are designed to illuminate the nature of the information that can be extracted from an extrafoveal object, retained across a saccade, and integrated with foveal information during a subsequent eye fixation.

The research reported here was supported by grants from the National Institute of Mental Health (R03 MH53023-01) and the U.S. Army Research Office (DAAH04-94-G-0404). This article was written while the first author (J.H.) was visiting the Laboratory of Experimental Psychology at the University of Leuven, and he thanks Professor Gery d'Ydewalle and the members of the laboratory for their hospitality. and Paul van Diepens, Peter DeGraef, and Karl Verfaillie in particular for many interesting discussions of the issues raised here. Thanks are also extended to Fernanda Ferreira for comments on an earlier draft of this article and to David Irwin and an anonymous reviewer for their helpful suggestions. Comments concerning this article should be addressed to J. M. Henderson. Department of Psychology, Michigan State University, East Lansing, MI 48824-1117 (e-mail: john@ eyelab.msu.edu)
For example, Pollatsek, Rayner, and Collins (1984) asked subjects to make an eye movement to a stimulus presented $5^{\circ}$ or $10^{\circ}$ to the left or right of fixation as soon as it appeared. The initial extrafoveal stimulus was replaced during the saccade (when vision is suppressed) with an object that the subject was to name as rapidly as possible following the saccade. In one condition, the initial stimulus was a preview of the object to be named; in another condition, an empty box was displayed instead. The main result was that naming latencies were markedly reduced when the object was previewed extrafoveally prior to the saccade compared with when it was not. These data suggest that a great deal of information can be acquired from an object $10^{\circ}$ from fixation in a dynamic viewing paradigm.

It could be argued that subjects in the Pollatsek et al. (1984) study extracted a relatively large amount of extrafoveal information only because a single object was presented in the display. It might be that in a more cluttered display, extrafoveal object information would no longer be acquired and integrated. Contrary to this hypothesis, large preview benefits can be observed in this paradigm when other objects are also present in the display. Henderson, Pollatsek, and Rayner (1987) presented an object at fixation in addition to the extrafoveal preview stimulus and again found large preview benefits at an eccentricity of $10^{\circ}$. Henderson, Pollatsek, and Rayner (1989) showed that the results from the naming paradigm generalize to ubject viewing over longer viewing periods and with multiple objects: Subjects viewed arrays of four line drawings of objects while their eye movements were recorded. The objects in the arrays were separated by about $4.5^{\circ}$. The subject's task was to examine each array in preparation for an immediate probe memory test asking whether a particular object had appeared in the immediately prior array. The primary dependent measure was fixation time 
on each object as a function of whether an extrafoveal preview was available prior to fixation. As in the objectnaming studies, large preview benefits were again observed, demonstrating that preview benefits can be generated over multiple fixation-saccade cycles and when multiple foveal and extrafoveal objects are present in the visual field. Overall, these experiments show that considerable information about an object can be acquired up to $10^{\circ}$ from fixation even when other objects appear in the visual field.

\section{Scene Perception and Object Identification}

Experiments that have monitored eye movements during scene perception provide an additional source of evidence concerning the role of foveal information in object identification. These studies have produced results suggesting that object identity and object detail are coded primarily when the eyes fixate directly or very near an object (Nelson \& Loftus, 1980; Parker, 1978). For example, Nelson and Loftus (1980) had subjects examine line drawings of scenes in preparation for a later recognition memory test. During the learning phase, the subject's eye movements were recorded. In the recognition phase, distractors were presented that had been constructed by changing one of the objects that had appeared in the original scene (e.g., changing a paint roller to a baseball bat). The subjects were most likely to detect the change (to say "no" to the recognition question) when they had fixated directly or very near the object during the learning phase. The likelihood of detecting the change decreased dramatically as a function of the distance of the closest fixation to the changed object. In one experiment, performance dropped to chance when no fixation landed closer than $1.8^{\circ}$ to the object. In a second experiment, performance was slightly above chance at $1.8^{\circ}$ and beyond. These results suggest that subjects had to fixate directly or near an object if they were going to encode that object into memory, although there was some suggestion that object information could be encoded from beyond the fovea.

In a similar study, Parker (1978) asked subjects to study line drawings of simple scenes in preparation for a recognition memory test. Parker was interested in the eye movements of the subjects as they examined the scenes during the test phase. The distractor scenes differed from the learned scenes by changes made in specific objects in the scenes (i.e., size changes, type and token substitutions, and deletions). Parker found that the subjects fixated 5.9 of the 6 objects on average in each scene, suggesting that foveal processing was necessary for the extraction of detailed object information. This conclusion is strengthened by the finding that the objects were almost always fixated despite their extreme familiarity: Each scene was viewed 60 times by each subject over the course of the experiment.

While the above free-viewing studies suggest that foveal processing is necessary for the acquisition of detailed object information, it is also clear that some amount of object information can be acquired from the periph- ery. In the study discussed above, Parker (1978) found that when an object had been deleted, subjects failed to fixate the blank space on $85 \%$ of the recognition trials, suggesting that deletions could be detected in the periphery. Parker also found that subjects tended to "skip ahead" to a changed object as they scanned the scene. Given that the nearest objects in Parker's scenes were separated by $6.5^{\circ}$ of visual angle (mean separation $=10^{\circ}$ ), these data suggest that some information about the changed object had been acquired in the periphery.

A number of other studies have shown that the eyes tend to be attracted to "informative" areas of a scene outside of the fovea (Antes, 1974; Mackworth \& Morandi, 1967; Loftus \& Mackworth, 1978). For example, Loftus and Mackworth monitored eye movements while subjects viewed line drawings of scenes. The subjects were asked to look at the scenes "as if they were going to have a later recognition test." There were two versions of each scene, one containing an informative object (an object that would not be expected in the scene) and the other containing an uninformative object (one that would be expected). For example, a farm scene contained either an octopus or a tractor at the same position. Informative objects were more likely to be fixated and were fixated earlier than uninformative objects. The average distance of the saccade to these objects was $6.5^{\circ}$ to $8^{\circ}$ of visual angle $\left(6.4^{\circ}\right.$ to $7.2^{\circ}$ when the object was fixated during the second fixation on the scene), indicating that object informativeness had been detected in the periphery.

The above scene-perception experiments suggest that subjects generally need to fixate directly or very close to an object in order to encode detailed information from it (Parker, 1978), although they can sometimes detect changes to objects that are not directly fixated (Nelson \& Loftus, 1980). At the same time, subjects can detect object deletions (Parker, 1978) and tend to fixate "informative" (Loftus \& Mackworth, 1978; see also Antes, 1974; Mackworth \& Morandi, 1967) or changed objects sooner (Parker, 1978), based on nonfoveal information. Overall, the data from scene-perception experiments suggest that some information may be extracted from objects beyond the fovea but that performance requiring encoding of object identity and detail improves markedly with foveal fixation.

\section{Present Study}

Both the transsaccadic integration and free sceneviewing studies reviewed above provide evidence that considerable information about object identities can be acquired from beyond the fovea. At the same time, the scene-perception studies suggest that foveal object processing may be necessary for complete object encoding. While these studies are suggestive, none of them included a direct manipulation of the presence of foveal information. The main purpose of the present study was to examine the degree to which foveal information was necessary for object encoding. This issue was investigated by using a contingent display-change paradigm to produce an artificial scotoma at the subject's fovea during object 
viewing. Subjects examined linear arrays of four line drawings of objects, as shown in Figure 1, while their eye movements were recorded. Three conditions were compared, one in which a central foveal scotoma was created, one in which a peripheral scotoma was created, and a control condition in which the scotoma was absent. The subject's task was to determine the identities of the four objects present in the currently viewed array. Immediately after each array was viewed, a word probe was presented to test the degree to which the subject had encoded those objects.

To investigate the effect of the loss of foveal information on object encoding, two general types of measures were examined, the accuracy of the subject's responses to the probes and the effects of the foveal scotoma on the subject's eye movements during object encoding. We generated three sets of predictions for these measures. First, if foveal information is necessary for object encoding, the presence of a central scotoma should lead to very poor (in the limit, chance) performance in the identification task. Eye-movement patterns should also be disrupted as the subject attempts to compensate for the loss of the foveal information. Second, if foveal information is unnecessary for identification but facilitates perceptual or memory encoding, accuracy should be relatively good whether a central scotoma is present or not, but eyemovement behavior in the foveal scotoma condition should be disrupted as the subject attempts to compensate for the loss of foveal information by increasing peripheral encoding. Third, if foveal information is completely unnecessary for the encoding of object identity, both accuracy and eye-movement behavior should be equivalent when foveal information is and is not present.

\section{EXPERIMENT 1}

In Experiment 1, subjects viewed arrays of line drawings of objects while their eye movements were recorded. The subject's task was to determine the identities of the objects in the current array and then to answer "yes" or "no" to an immediately following test-probe word. For example, the subject would view an array like that shown in Figure 1, and then answer "yes" if the probe word was "chicken" and "no" if it was "plane." On one third of the trials, all of the objects were visible throughout the trial; this was the scotoma-absent condition. On another third of the trials, the information contained in the currently fixated region of the display was removed from the display. Thus, in this scotoma-centered condition, visual information that would normally be projected to the highest acuity region of the retina (the fovea) was not present. On the final third of the trials, the information contained in the region immediately to the right of the currently fixated region was not present; this was the scotoma-offset condition.

To generate the moving scotoma, we used a boundary technique like that employed by Henderson et al. (1989). A software-defined invisible boundary was placed halfway between each stimulus, dividing the display into six equal regions. As long as the eyes remained in one region, the display did not change. When the eyes crossed a boundary dividing two regions, the display changed in a particular way, depending on the condition. For example, when the eyes crossed into Region 2 in the scotoma-centered condition, the object occupying Region 2 was removed. These display changes took place during the saccade from one stimulus to another so that they were accomplished while vision was suppressed.

\section{Method}

Subjects. Twelve members of the Michigan State University subject pool (undergraduate students, 7 males and 5 females), all of whom had normal, uncorrected vision, participated either for course credit or for pay. Some of the subjects had had experience with eye-movement experiments, but none had participated in an artificial scotoma experiment and all were naive with respect to the hypotheses under investigation.

Stimuli. The stimuli were simple linear arrays of line drawings. Each array contained an initial and a final marker pattern surrounding pictures of 4 real-world objects, as shown in Figure 1. Ninetysix arrays were created by combining 192 objects. Each of the first 48 arrays was constructed by randomly selecting 4 objects without replacement from the entire set and then randomly placing those objects into each of the four central array positions. Each of the second 48 arrays was created in the same way, with the constraint that no object could appear twice in the same array position. In total, then, 96 arrays were created such that each of the $192 \mathrm{ob}-$ jects appeared exactly twice across arrays but in a different array position across repetitions.

The objects used in the arrays were taken from Snodgrass and Vanderwart (1980). These objects were digitized using a HewlettPackard Scanjet IIc flatbed scanner, and stray pixels were removed as necessary, using Adobe Photoshop and Aldus Photostyler. Each object included in the experiment had a name-agreement score greater than $50 \%$ and a visual complexity score greater than 1.50 , according to the Snodgrass and Vanderwart ( 1980 ) norms. In addition, the objects had to be of good quality following digitization, as judged by the experimenters. The beginning and ending marker was a circle containing a large cross and two concentric circles (see Figure 1). These markers were included so that the first and last object would be laterally masked on both sides (as were the two central objects) and so that the scotoma-centered condition would he defined when fixation was at the beginning position in an array and the scotoma-offset condition would be defined when fixation was on the last object in an array.
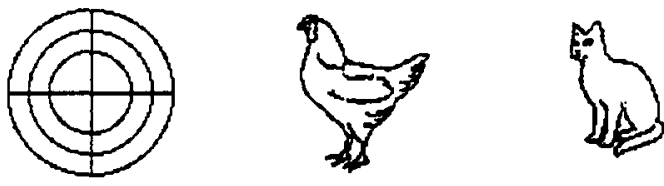

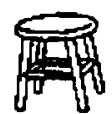

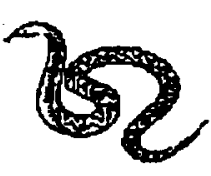

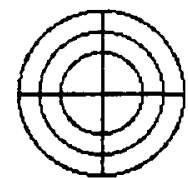

Figure 1. An example of the object arrays shown in Experiments 1 and 2. 
At the employed viewing distance of $1 \mathrm{~m}$, the display area subtended $15.3^{\circ}$ of arc horizontally and $12.0^{\circ}$ vertically. Within that area, the arrays occupied a central strip subtending $13.5^{\circ}$ horizontally. The largest object was $1.9^{\circ}$ in diameter; the average object and the markers were $1.5^{\circ}$ wide and $1.5^{\circ}$ in height. There were about $2.4^{\circ}$ between the centers of neighboring objects. The diameter of the scotoma was effectively the size of the region.

Word test probes were names of common objects and were paired with an object array. Fifty percent of these words were used for the "yes" responses and named an object in its paired array. The other $50 \%$ of the word probes named objects that were not included in the experiment; these distractor probes were taken from the same class as those used as "yes" probes (most were taken from Snodgrass and Vanderwart, 1980, and were objects that were not used in the experiment). Probe words, which ranged from $1^{\circ}$ to $5^{\circ}$ across the set, were shown at the center of the display monitor in an Arial font at about three characters per degree.

Apparatus. The stimuli were displayed at a resolution of $800 \times$ 600 pixels on an NEC Multisync XE 15 -in. monitor driven by a Hercules Dynamite Pro super videographics adapter (SVGA) card. The screen refresh rate was $100 \mathrm{~Hz}$. The contours of the objects and markers appeared black (pixels off) against a gray (pixels on) background (the gray was created by setting the red, green, and blue channels to an intensity value of 16 , where white has an intensity value of 64 on each channel). The scotoma was displayed by replacing the object and marker contours with the gray background color. The time needed to fully replace a stimulus with the background (the "decay rate" of the stimulus) was one raster scan. Display luminance was generally low and was individually adjusted to a comfortable level for each subject. The room was otherwise illuminated by a low-intensity, indirect light source.

Eye movements were monitored using a Generation 5.5 Stanford Research Institute Dual Purkinje Image Eyetracker (Crane, 1994; Crane \& Steele, 1985), which has a resolution of $1 \mathrm{~min}$ of arc and a linear output over the range of the visual display used. A bite-bar and forehead rest were used to maintain the subject's viewing position and distance. The position of the right eye was tracked, although viewing was binocular. Signals were sampled from the eyetracker, using the polling mode of the Data Translations DT2802 analog-to-digital converter. This method produced a sampling rate of better than 1 sample per millisecond.

The scotoma condition was defined by eye position within one of six regions: Each region was defined by two vertical boundaries (for the objects) or one boundary and an edge of the display area (for the markers). The boundaries divided the display into six equal regions, with each boundary placed exactly intermediate between two stimuli. So long as the line of regard was within two boundaries defining a region, the image for that region in that scotoma condition was displayed. As soon as the line of regard was detected in a new region, a display change was initiated. In the scotoma-centered condition, whenever the eyes crossed one of the two boundaries defining a particular region, a video page was presented that contained an image of all of the stimuli (objects and markers) except for the stimulus occupying the current region. In the scotoma-offset condition, whenever the eyes crossed one of the two boundaries defining a particular region, a video page was presented that contained an image of all of the stimuli (objects and markers) except for the stimulus occupying the position immediately to the right of the current region. In the scotoma-absent condition, the display changed from a video page containing an image of the complete array to another page containing the same image. These display changes required an average of $5.84 \mathrm{msec}$ (minimum $=1.683 \mathrm{msec}$, maximum $=10 \mathrm{msec}$ ) and were therefore completed during saccades between regions. In the scotoma-absent condition, exactly the same type of display change (i.e., page changing) took place as in the two scotoma-present conditions so that any effect of the change itself would be controlled.
Buttonpress responses were collected using a button panel connected to a dedicated input-output $(\mathrm{I} / \mathrm{O})$ card; depressing a button stopped a millisecond clock on the I/O card and generated a system interrupt that was serviced by software. The eyetracker, display monitor, and $\mathrm{I} / \mathrm{O}$ card were interfaced with a microcomputer running a $66-\mathrm{MHz} 486 \mathrm{DX} 2$ processor. The computer controlled the experiment and maintained a complete eye-movement and buttonpress record for each trial.

Procedure. Subjects first read a description of the experiment along with a set of instructions. The bite-bar was then prepared, and the experimenter orally reviewed the task instructions and answered any questions. The eye-tracking apparatus was then calibrated. Calibration consisted of having the subject fixate four calibration markers at the top, bottom, left, and right sides of the display area. Calibration was checked by displaying a calibration screen consisting of six test positions and a fixation marker that indicated the computer's estimate of the current fixation position. The subject fixated the test positions, and if the fixation marker was $\pm 5 \mathrm{~min}$ of arc from each, calibration was considered accurate.

After the calibration, the subject saw 12 practice trials, 4 of each scotoma condition presented in a random order. Following the practice trials, any remaining questions were answered, and the subject then took part in 96 experimental trials. The subjects were given an enforced break about halfway through the experimental trials, and were allowed to take additional breaks following any trial, although most did not exercise this option.

A trial consisted of the following events. First, the calibration screen was shown and calibration was checked. The eyetracker was recalibrated whenever calibration was deemed inaccurate using the $\pm 5 \mathrm{~min}$ of arc criterion. Following the calibration check, the subject fixated the leftmost marker to indicate that he or she was ready for the trial to begin. The experimenter then started the trial: The fixation display was replaced by the trial display containing a linear array of two markers and four objects. The subject looked through the array to identify the four objects, and then terminated the display by manually pressing either of two response buttons. The button response caused offset of the object array and immediate onset (within the limitations of the screen refresh rate) of the probe-word display. The probe word named one of the four objects in the array $50 \%$ of the time and named an object not included in the experiment the other $50 \%$ of the time. For the "yes" probe trials, each of the four object positions was probed an equal number of times. The subject pressed the left button on the response panel to indicate that the probe had been contained in the array, and the right button to indicate that it had not. The probe remained on the screen until the subject responded. Following this response, the calibration screen reappeared for about $6 \mathrm{sec}$ while the images for the next trial loaded into video memory.

The factorial combination of three scotoma conditions (scotoma absent, scotoma centered, and scotoma offset) and two probe conditions ("yes" and "no" trials) created six experimental conditions. Each subject participated in 96 experimental trials, 16 trials in each of these six conditions (i.e., 32 trials in each scotoma condition). Six stimulus lists were created such that each object array appeared once in each list and each array appeared in all six conditions across lists. Two subjects received each of the six lists. The order of array presentation (and hence condition presentation) was determined randomly for each subject. The entire experiment lasted approximately $45 \mathrm{~min}$.

\section{Results}

Eye-movement data analysis. Raw-data files consisted of time and position values for each eyetracker sample. Because the analyses of interest are concerned with fixations, the saccades were removed from the data. Saccades were defined as velocities greater than $6.58^{\circ}$ per sec- 
ond. Manual inspection of the raw data files confirmed that this criterion was more effective at eliminating the initial and final stages of a saccade than were criteria of greater velocity. Once saccades had been eliminated, fixation positions and durations were computed over the remaining data. Fixation positions and durations were initially computed independently of the positions of the objects. The duration of a fixation was the elapsed time between two consecutive saccades. During a fixation, the eyes often drift. The position for a given fixation was taken to be the mean of the position samples (in pixel values) taken during that fixation weighted by the durations of each of those position samples, as given by the following equations, where $x \operatorname{pos}_{\mathrm{fix}}$ and $y$ pos $_{\mathrm{fix}}$ are the $x$ and $y$ positions of the fixation, $x$ pos $_{\text {sample }}$ and $y$ pos sample are the $x$ and $y$ positions of the current sample, and duration sample is the duration of the current sample:

$$
\begin{aligned}
x \operatorname{pos}_{\mathrm{fix}} & =\frac{\sum\left(x \operatorname{pos}_{\text {sample }} \times \text { duration }_{\text {sample }}\right)}{\sum \text { duration }_{\text {sample }}} \\
y \operatorname{pos}_{\mathrm{fix}} & =\frac{\sum\left(y \text { pos }_{\text {sample }} \times \text { duration }_{\text {sample }}\right)}{\sum \text { duration }_{\text {sample }}} .
\end{aligned}
$$

Each fixation was then assigned to an object or marker on the basis of its $x$ and $y$ position values. Scoring regions for the four objects and the two markers were defined by dividing the display area into six equal vertical strips with the stimuli centered in each strip. Each scoring region was the same as the boundary region as defined for the scotoma conditions. A fixation was counted as belonging to the stimulus (object or marker) occupying the same vertical strip as that fixation.

For the analyses reported below, the focus was on eyemovement patterns over the four object drawings in the center of each array as a function of scotoma condition. Analyses of variance (ANOVA) were conducted for each measure with ordinal object position (for the four objects) and scotoma condition as within-subject factors. The data were collapsed over response condition ("yes" or "no"), because these trials were equivalent during array viewing (i.e., subjects could not know during array viewing which type of question they would receive after array offset). Eye-movement behavior on the markers was ignored in the statistical treatment although, for completeness, we include the data from the markers in the figures. We note that the data shown in the figures for the markers must be treated cautiously: Subjects began each trial by fixating the initial marker, and there were very few saccades back into that region. Also, the subjects did not spend much time on either marker and fixated the final marker on fewer than $10 \%$ of the trials, so the means for those regions were based on very few observations.

Performance accuracy. Subjects were able to determine with a high degree of accuracy whether the probed object was present or absent across all three scotoma conditions. Table 1 presents the mean proportions of hits $[p(\mathrm{~h})]$ and false alarms $[p(\mathrm{fa})]$ as a function of scotoma
Table 1

Mean Hit Rate and False-Alarm Rate as a Function of Response Condition and Scotoma Condition, Experiment 1

\begin{tabular}{lcccc}
\hline & \multicolumn{3}{c}{ Scotoma Condition } & \\
\cline { 2 - 4 } & Absent & Offset & Centered & Mean \\
\hline$p(\mathrm{~h})$ & .952 & .943 & .926 & .940 \\
$p(\mathrm{fa})$ & .021 & .016 & .021 & .019 \\
\hline
\end{tabular}

condition. The overall hit rate was .940 , and the overall false-alarm rate was .019 . The hit rate was slightly lower in the scotoma-centered condition than in the scotomaabsent condition (.026 difference), while the false-alarm rates for these conditions were equivalent. Statistically, performance did not differ as a function of scotoma condition, either for $p(\mathrm{~h})\left(F<1, M S_{\mathrm{e}}=.0052\right)$ or for $p(\mathrm{fa})$ $\left(F<1, M S_{\mathrm{e}}=.0008\right)$.

\section{Global Measures of Eye-Movement Patterns}

To determine whether the presence of either the central or the offset scotoma had any influence on the difficulty with which the objects were encoded, we examined four global measures of the eye movements that the subjects made as they examined the arrays. These measures were the spatial distribution of processing time, the number of entries into each region, the total amount of time spent in each region, and the total number of individual fixations made in each region.

Spatial distribution of processing time. To determine how object processing time was distributed over the arrays in the three scotoma conditions, we computed the total viewing time on the displays as a function of horizontal position, collapsed across vertical position. These data for each of the scotoma conditions are plotted in Figure 2. As can be seen, subjects tended to spend the majority of processing time on the objects rather than on the background space between them, with the majority of time concentrated on the center of each object in all conditions. Interestingly, the distributions observed in the scotoma-centered condition were similar to those in the other scotoma conditions, suggesting that subjects were moving their eyes to the centers of the objects in the scotoma-centered condition even though the center of the object would disappear once the eyes landed there. At the same time, there was a tendency, apparent in Figure 2 , for the eyes to fixate the regions between objects for a longer time in the scotoma-centered condition than in the scotoma-offset and scotoma-absent conditions. We quantified this tendency by defining a region between each pair of objects and summing the fixation times in these three regions. These regions were 26 pixels wide and display-area height. Total fixation times in these intermediate regions were reliably longer in the scotomacentered condition $(291 \mathrm{msec})$ than in the scotomaabsent (151 msec) and scotoma-offset (172 $\mathrm{msec})$ conditions $\left[F(2,22)=6.863, M S_{\mathrm{e}}=50053, p<.01\right]$.

We computed similar fixation-time plots for the proportion of total viewing time on the displays as a function of vertical display position, collapsed across horizontal 

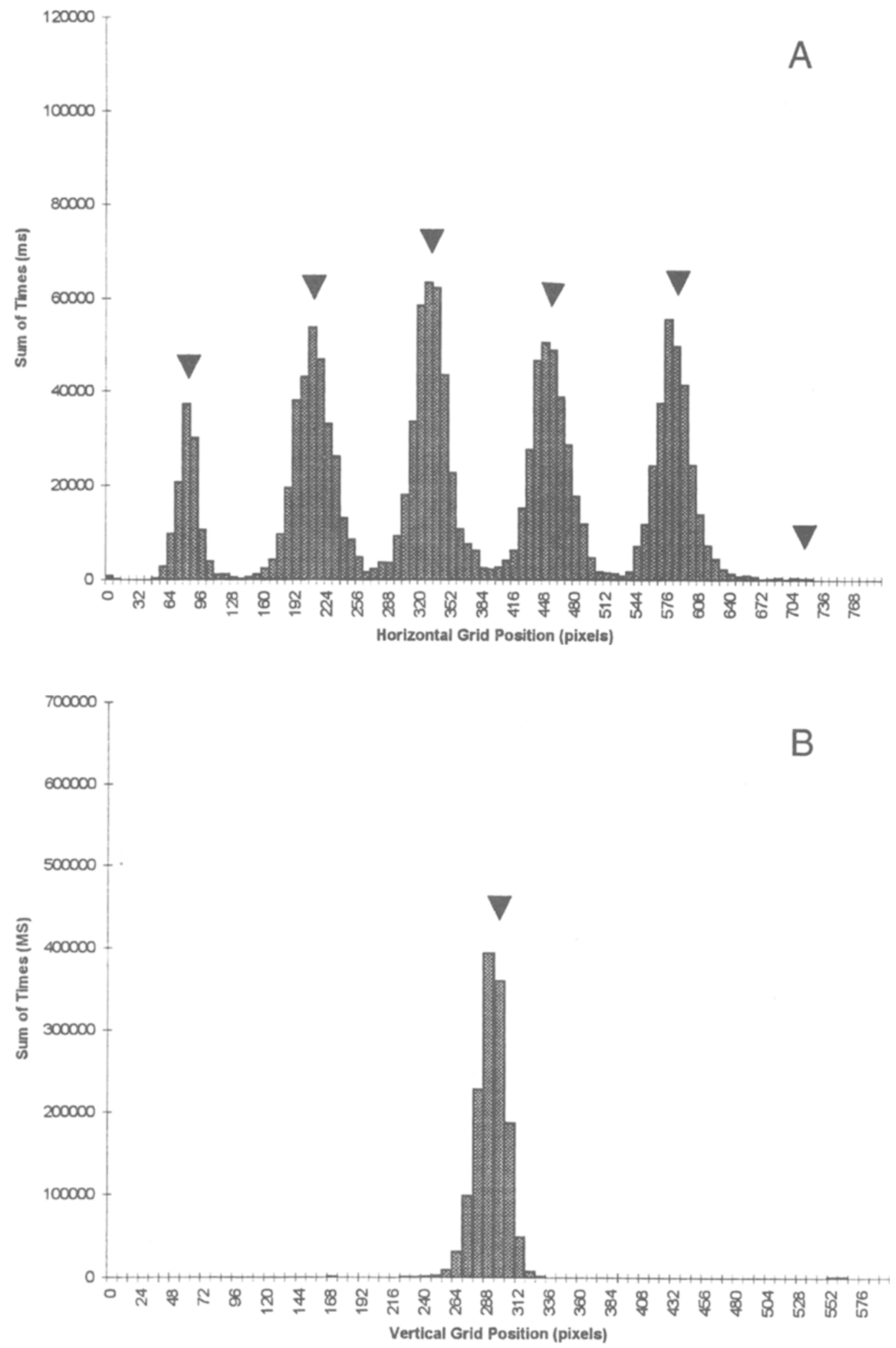

Figure 2. Total viewing time on the arrays as a function of position (in screen pixel coordinates), Experiment 1. Panel A shows horizontal position collapsed over vertical position, and panel B shows vertical position collapsed over horizontal position, for the scotoma-absent condition. Arrows in each panel show the centers of the six regions. (See following pages for equivalent plots for the scotoma-offset condition Ipanels $\mathrm{C}$ and $D \mid$ and the scotoma-centered condition (panels $E$ and $F \mid$.) 

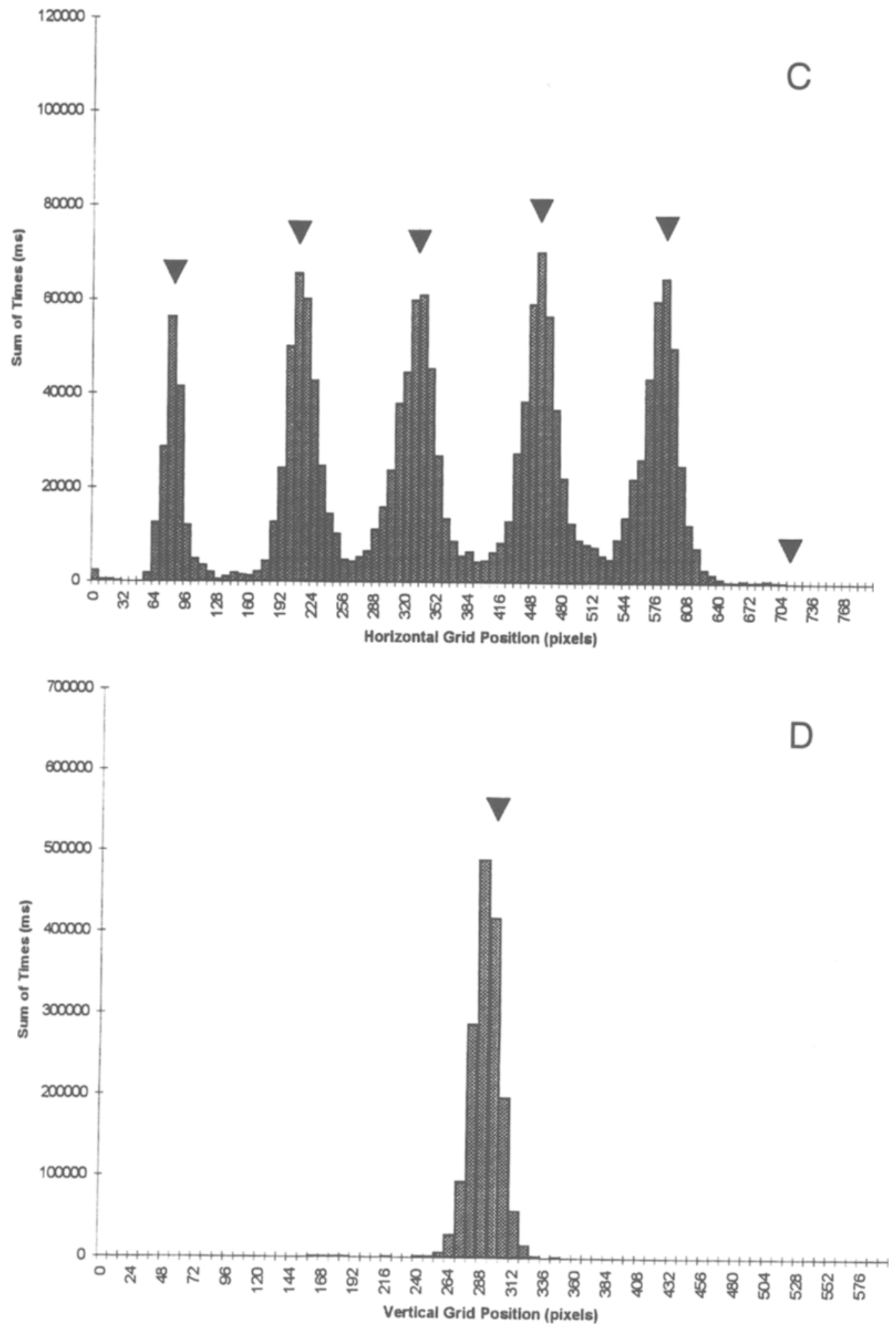

Figure 2 (Continued). Total viewing time on the arrays as a function of position (in screen pixel coordinates), Experiment 1. Panel $C$ shows horizontal position collapsed over vertical position, and panel $D$ shows vertical position collapsed over horizontal position, for the scotoma-offset condition. Arrows in each panel show the centers of the six regions. (See following page for equivalent plots for the scotoma-centered condition [panels $E$ and $F$ ] and previous page for the scotoma-absent condition (panels $A$ and $B$.) 

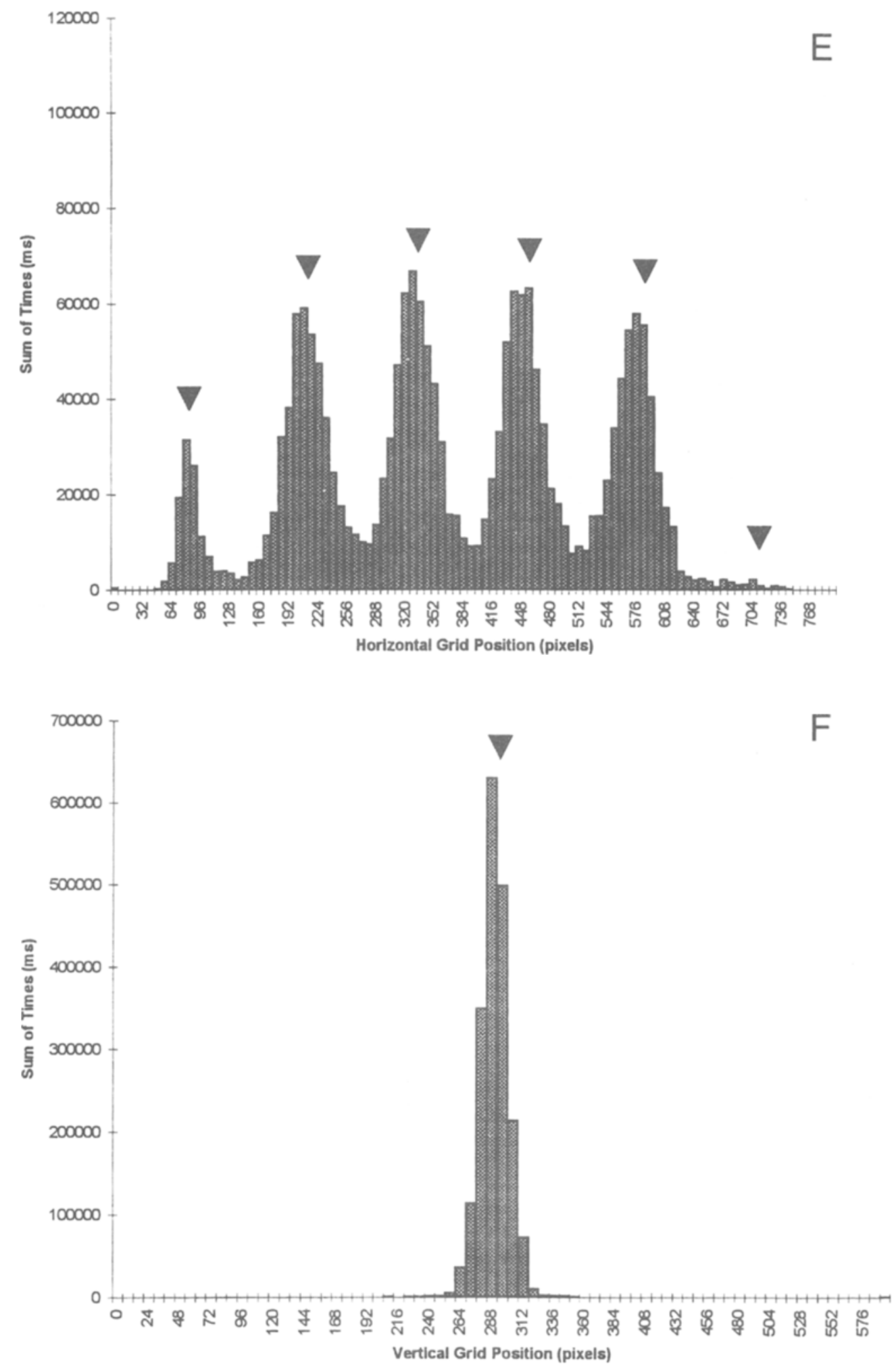

Figure 2 (Continued). Total viewing time on the arrays as a function of position (in screen pixel coordinates), Experiment 1. Panel $E$ shows horizontal position collapsed over vertical position, and panel $F$ shows vertical position collapsed over horizontal position, for the scotoma-centered condition. Arrows in each panel show the centers of the six regions. (See preceding pages for equivalent plots for the scotoma-absent condition [panels $A$ and $B$ ] and the scotoma-offset condition (panels $C$ and $D \mid$.) 


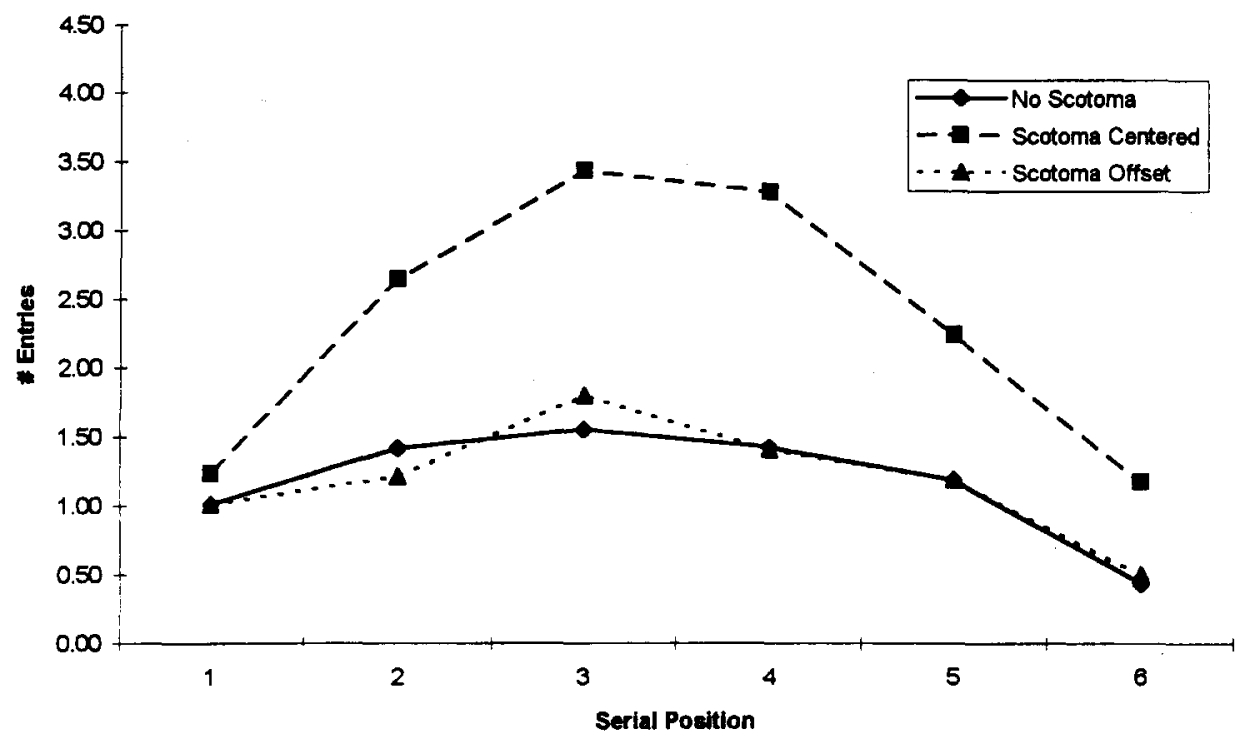

Figure 3. Mean number of entries into the six regions of the arrays as a function of ordinal region position and scotoma condition, Experiment 1.

position. These data are also shown in Figure 2. Again, the majority of time was spent on the centers of the objects. Overall, the spatial distribution of fixation time is similar to that reported by Henderson (1993), showing that during visual object processing fixations are concentrated on objects rather than on background and on the centers of objects rather than on their boundaries.

Number of entries. Number of entries was defined as the number of times that the eyes entered each of the regions following a saccade that originated from a launch position beyond that region. (The initial fixation on the first marker was counted as an entry into that region.) This measure serves as an index of the degree to which the eyes moved from object to object. Figure 3 shows the mean number of entries into each region as a function of ordinal region position and scotoma condition, and Table 2 shows the mean number of entries into the four object regions as a function of scotoma condition, collapsed over region. Overall, subjects entered each object region 1.90 times. The number of entries in the scotoma-absent and scotoma-offset conditions was equivalent, while there were more entries in the scotoma-centered condition, leading to a main effect of scotoma condition $[F(2,22)=$ $\left.35.97, M S_{\mathrm{e}}=1.470, p<.001\right]$. This effect was exaggerated for the central two object positions as compared with the first and last object positions, leading to a reliable interaction of scotoma condition and ordinal position $\left[F(6,66)=5.444, M S_{\mathrm{e}}=.1240, p<.001\right]$.

Total fixation time. Total fixation time was defined as the total amount of time spent fixating each region during array viewing. If the amount of time the eyes spend looking at an object is taken to indicate object encoding time (De Graef, Christiaens, \& d'Ydewalle, 1990; Henderson et al., 1987; Loftus \& Mackworth, 1978), then total fixation time is the total encoding time in a region. Figure 4 (top panel) shows the mean total fixation time in each region as a function of ordinal region position and scotoma condition, and Table 2 shows the mean total fixation time for the four object regions, as a function of scotoma condition, collapsed over region. Overall, the mean total fixation time on each object region was $977 \mathrm{msec}$. Total fixation time was reliably affected by scotoma condition $\left[F(2,22)=15.60, M S_{\mathrm{e}}=119,887, p<.001\right]$, with longest total times in the scotoma-centered condition, intermediate times in the scotoma-offset condition, and shortest total times in the scotoma-absent condition. The

Table 2

Mean Number of Entries, Mean Total Fixation Time (in Milliseconds),

Mean Total Fixation Count, Mean Gaze Duration (in Milliseconds), and Mean Gaze Fixation Count for the Four Line Drawings, Collapsed Over Ordinal Position, Experiment 1

\begin{tabular}{lccccc}
\hline $\begin{array}{c}\text { Scotoma } \\
\text { Condition }\end{array}$ & $\begin{array}{c}\text { Number of } \\
\text { Entries }\end{array}$ & $\begin{array}{c}\text { Total } \\
\text { Fixation Time }\end{array}$ & $\begin{array}{c}\text { Total } \\
\text { Fixation Count }\end{array}$ & $\begin{array}{c}\text { Gaze } \\
\text { Duration }\end{array}$ & $\begin{array}{c}\text { Gaze } \\
\text { Fixation Count }\end{array}$ \\
\hline Absent & 1.39 & 814 & 2.50 & 634 & 1.93 \\
Offset & 1.40 & 920 & 2.63 & 677 & 1.94 \\
Centered & 2.90 & 1.196 & 3.57 & 406 & 1.20 \\
Mean & 1.90 & 977 & 2.90 & 573 & 1.69 \\
\hline
\end{tabular}



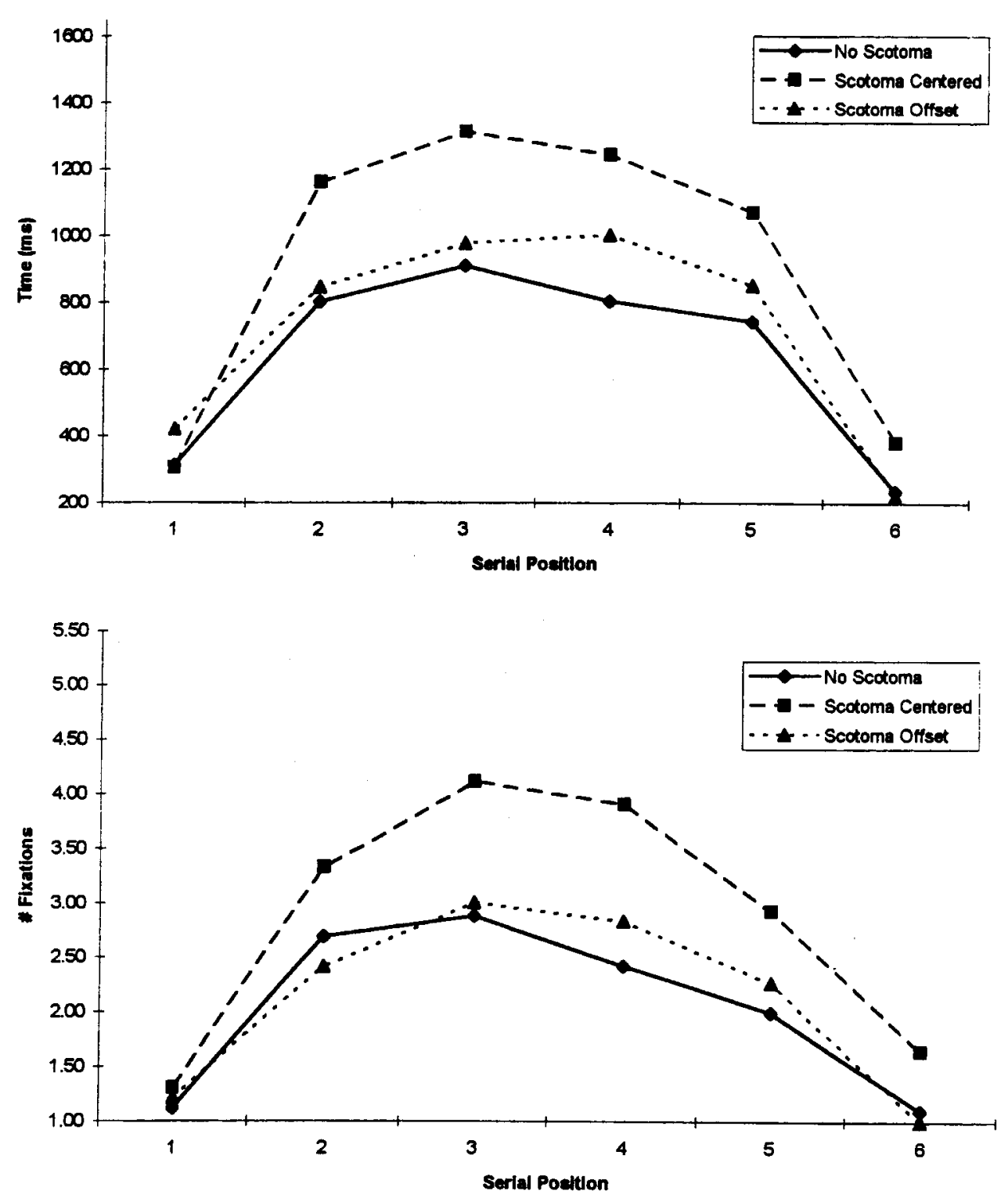

Figure 4. Mean total fixation time in milliseconds (top panel) and mean total fixation count (bottom panel) on the arrays as a function of ordinal region position and scotoma condition, Experiment 1.

effect of the scotoma condition was not mediated by ordinal region position $(F<1)$. The tendency, apparent in Figure 4 , for total time to be longer on the central two object positions than on the first and fourth object positions was reliable, as shown by a main effect of ordinal object position $\left[F(3,33)=6.919, M S_{\mathrm{c}}=32,534, p<.005\right]$.

Total fixation count. Total fixation count was defined as the total number of discrete fixations in a region. Figure 4 (bottom panel) shows the mean total fixation count for each region as a function of ordinal position and scotoma condition, and Table 2 shows the mean total fixation count for the four object regions as a function of scotoma condition, collapsed over region. Overall, the mean total fixation count on each object region was 2.90 . These data showed a pattern similar to that of the total fixation time data, with a greater number of fixations in the scotoma-centered condition, an intermediate number in the scotoma-offset condition, and the fewest in the scotoma-absent condition $\left[F(2,22)=16.59, M S_{\mathrm{e}}=\right.$ $1.9093, p<.005]$. There were also more fixations on the center two object positions, leading to a main effect of ordinal object position $\left[F(3,33)=5.648, M S_{\mathrm{e}}=.2869, p<\right.$ $.001]$. The two factors did not reliably interact $[F(6,66)=$ $\left.1.641, M S_{\mathrm{e}}=.3238, p>.10\right]$.

\section{Local Measures of Eye-Movement Patterns}

To determine how quickly the scotoma affected eyemovement patterns, we examined two local measures of eye-movement behavior on the arrays. These measures were gaze duration and gaze fixation count. ${ }^{2}$ 

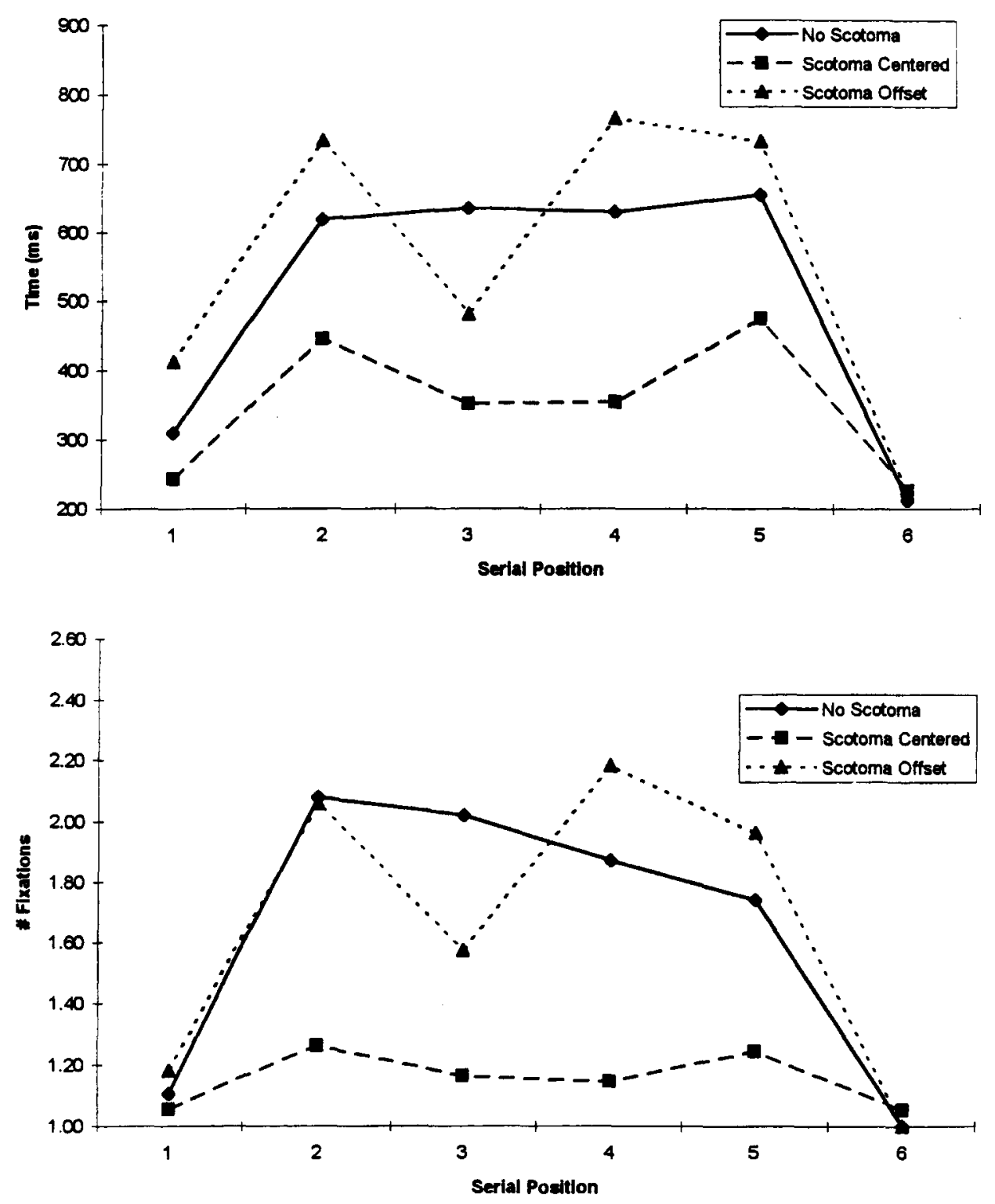

Figure 5. Mean gaze duration in milliseconds (top panel) and mean gaze fixation count (bottom panel) on the arrays as a function of ordinal region position and scotoma condition, Experiment 1.

Gaze duration. Gaze duration was defined as the sum of all fixation durations between first entry and first exit in a region. Gaze duration has often been taken as a measure of object encoding time in scene-perception research (e.g., Antes, 1974; Friedman, 1979; Loftus \& Mackworth, 1978).

Figure 5 presents mean gaze duration in each region as a function of ordinal position and scotoma condition, and Table 2 shows the mean gaze duration on the four object regions as a function of scotoma condition, collapsed over region. Overall mean gaze duration in each object region was $573 \mathrm{msec}$. The scotoma-centered condition produced shorter gaze durations than did the scotomaabsent condition, and the scotoma-offset condition pro- duced the longest gaze durations $\left[F(2,22)=15.59, M S_{\mathrm{e}}=\right.$ $65,369, p<.001]$. This pattern held for three of the four ordinal object positions $(1,3$, and 4$)$, but differed for Position 2, leading to a reliable interaction of scotoma condition and ordinal position $\left[F(6,66)=5.912, M S_{\mathrm{e}}=\right.$ $11,734, p<.001]$.

Gaze fixation count. Gaze fixation count was defined as the number of individual fixations between first entry and first exit for that region. This count is the number of fixations whose durations were summed to produce the gaze duration. Figure 5 (bottom panel) presents the mean gaze fixation count in each region as a function of ordinal region position and scotoma condition, and Table 2 shows the mean gaze fixation count on the four 
object regions as a function of scotoma condition, collapsed over region. Mean gaze fixation count in each object region was 1.69. The gaze fixation count was similar in the scotoma-absent and scotoma-offset conditions, with more fixations in those conditions than in the scotoma-centered condition $\left[F(2,22)=26.49, M S_{\mathrm{e}}=\right.$ $.3253, p<.001]$. The reduced number of gaze fixations at Position 2 in the scotoma-offset condition led to an interaction of scotoma condition $\times$ ordinal position $\left[F(6,66)=6.426, M S_{\mathrm{e}}=.0645, p<.001\right]$. At this point, it is not clear what to make of the drop in gaze duration and gaze fixation count at Position 2; as will be seen, in Experiment 2, we replicate the ordering of the three scotoma conditions but not this drop in the scotoma-offset condition at Position 2.

\section{Discussion}

In Experiment 1, we examined the degree to which object identification would be disrupted when central visual information was eliminated through the introduction of an artificial scotoma. The high level of accuracy to the test probe showed that subjects were able to identify the objects in all of the scotoma conditions, including the scotoma-centered condition. Performance in the probe memory task was very good in all conditions, with a modest and nonsignificant $2.6 \%$ decrease for the hit rates in the central-scotoma condition in comparison to the control condition and no difference at all in false-alarm rates. Thus, it is clear from this experiment that under the viewing conditions we employed (relatively small objects that were relatively close together in the visual field), central foveal information is not necessary for object identification. At the same time, however, viewing behavior changed in regular ways when foveal information was absent, as seen in the eye-movement measures. For example, the global eye-movement measures showed that a central scotoma led to more entries into each object region, greater total fixation time in each object region, and more individual fixations in each object region than did the scotoma-offset and scotoma-absent conditions. Similarly, the local eye-movement measures showed that viewing behavior was affected by the presence of a central scotoma at the earliest moments of viewing. Upon first encountering each object, the central scotoma led to shorter gaze durations and fewer gaze fixations than did the scotoma-offset and scotoma-absent conditions. The latter pattern of data suggests that the absence of foveal information leads the eyes to move along to a new (currently extrafoveal) source of information as quickly as possible. One question to be addressed in Experiment 2 was whether it was the absence of any type of foveal stimulation or the absence of meaningful object information that caused this effect.

The peripheral scotoma also caused some change in viewing behavior, as compared with the control condition, although not all measures showed these changes and, in general, the changes were smaller than those in the scotoma-centered condition. In the global measures, the offset scotoma led to the same number of entries into each object region as did the control condition, but more total time was spent and more individual fixations were made in each region when the offset scotoma was present than when it was not. In the local measures, the same number of gaze fixations were made whether an offset scotoma was present or not, but the gaze durations were longer for the scotoma-offset condition. Thus, the presence of an offset scotoma did produce some change to viewing patterns, but not all aspects of eye-movement behavior were affected, and those that were did not show nearly the same degree of disruption as was found in the central-scotoma condition.

In summary, the data from Experiment 1 are most consistent with the hypothesis that foveal information is beneficial, but not necessary, for object encoding during free viewing: Object identification was quite good when foveal information was not available, but eye-movement patterns suggested that more peripheral processing was necessary to overcome the loss of foveal information.

\section{EXPERIMENT 2}

In Experiment 1, we found that global viewing time increased but initial viewing time decreased when foveal information was absent. We have suggested that these viewing patterns were observed because useful object information was available only extrafoveally in the centralscotoma condition. This visual encoding hypothesis accounts for the greater total time taken to view the objects in the scotoma-centered condition (as reflected by total fixation time, total fixation count, and number of entries) by assuming that extrafoveal object encoding required more time than did foveal encoding, and accounts for shorter time in initial fixations (as reflected by shorter gaze durations and fewer gaze fixations) by assuming that, because attention must be devoted to the objects beyond the fovea in order to process them, the eyes tended to be drawn to those objects more quickly than when foveal information was present and attention was directed to that region.

An alternative explanation for the change in viewing behavior in the scotoma-centered condition is that the oculomotor system was directly affected by the absence of visual stimulation at the foveae. According to this oculomotor hypothesis, the absence of a stimulus at fixation caused both shorter time in individual fixations and greater total viewing time on the arrays, because the eyes were drawn to the extrafoveal stimuli in the absence of a foveal stimulus with which to engage a fixation response. In other words, the oculomotor hypothesis accounts for the longer overall encoding time in the scotoma-centered condition by positing that saccades with short latencies were generated in the absence of a fixation stimulus. This type of explanation gains plausibility from the finding that the offset of a foveal stimulus can lead to very brief fixations in a saccade-generation ("express saccade") task (Fischer \& Ramsperger, 1984; Fischer \& Weber, 1993). On this hypothesis, because fixations are abnormally short and any extrafoveal processing that 
might be taking place is interrupted by the onset of the premature saccade, more overall time would be required to encode the objects in the displays. In contrast, the visual encoding hypothesis reverses the direction of causality: Longer overall times are observed in the scotomacentered condition because extrafoveal object encoding is less efficient than foveal object encoding, and shorter gaze durations are observed because the act of attending to extrafoveal information tends to pull the eyes toward the attended object (Henderson, 1992b).

To determine whether the changed eye-movement patterns in the scotoma-centered condition were due to the lack of foveal stimulation, as proposed in the oculomotor hypothesis, rather than the lack of meaningful visual information at the fovea, as proposed in the visual encoding hypothesis, we replicated Experiment 1 with a change to the nature of the display shown in place of the object in the scotoma conditions. Instead of replacing the removed object with nothing (or, more precisely, with the gray background field), we replaced the object with a placeholder. This placeholder was a circle with a plus sign at the center. The diameter of the circle was equal to that of the average object. If the decreases in gaze duration and gaze fixation count and the increases in number of entries, total time, and total fixation count observed in the scotoma-centered condition of Experiment 1 were due to a disruption in eye-movement control brought about by the lack of foveal stimulation, then those decreases should not be observed in Experiment 2. If, on the other hand, those decreases were due to the loss of useful foveal information with which to identify objects, then eyemovement patterns observed in Experiment 2 should be similar to those observed in Experiment 1.

\section{Method}

Subjects. Twelve members of the Michigan State University subject pool (undergraduate students, 2 males and 10 females) participated in the experiment for course credit or for pay. These subjects had the same characteristics as in Experiment 1. There was no overlap in the individuals who participated in the two experiments.

Stimuli and Apparatus. The stimuli and apparatus were identical to those in Experiment 1, with the exception that a placeholder was used to replace the relevant object in the two scotoma conditions. This placeholder was $1.5^{\circ}$ in diameter and contained a centered plus sign that was about $.3^{\circ}$ horizontally and vertically.

Procedure. The procedure was identical to that of Experiment 1.

\section{Results}

Eye-movement data analysis. The data were treated as described in Experiment 1. The focus of the analyses was again on eye-movement behavior over the four objects as a function of scotoma condition. Eye-movement behavior on the markers was ignored in the statistical treatment, although, for completeness, we include the data from the markers in the figures, keeping in mind the caveats outlined in Experiment 1.

Performance accuracy. Table 3 presents the mean proportions of hits $[p(\mathrm{~h})]$ and false alarms $[p(\mathrm{fa})]$ as a function of scotoma condition. The subjects were able to
Table 3

Mean Hit Rate and False-Alarm Rate as a Function of Response Condition and Scotoma Condition, Experiment 2

\begin{tabular}{lcccc}
\hline & \multicolumn{3}{c}{ Scotoma Condition } & \\
\cline { 2 - 5 } & Absent & Offset & Centered & Mean \\
\hline$p(\mathrm{~h})$ & .951 & .968 & .846 & .921 \\
$p(\mathrm{fa})$ & .021 & .021 & .042 & .028 \\
\hline
\end{tabular}

determine, with a high degree of accuracy, whether the probed object was present or absent across all three scotoma conditions. The overall hit rate was .921 and the overall false-alarm rate was .028 . In contrast to Experiment 1 , however, the hit rate was reliably lower in the scotoma-centered condition than in the scotoma-absent and scotoma-offset conditions $\left[F(2,22)=7.729, M S_{\mathrm{e}}=\right.$ $.0068, p<.005]$. The difference in the hit rates between the scotoma-absent and scotoma-centered conditions was .105. There was also a tendency for the false-alarm rate to be slightly higher in the scotoma-centered condition than in the scotoma-absent and scotoma-offset conditions (.021 difference), but this difference was not reliable $\left[F(2,22)=1.195, M S_{\mathrm{e}}=.0015, p>.30\right]$. Overall, then, although reduced, performance was still quite good ( .85 hit rate and .04 false-alarm rate) when a central scotoma was present.

\section{Global Measures of Eye-Movement Patterns}

Spatial distribution of processing time. The total viewing time on the displays as a function of horizontal display position, collapsed across vertical position, for each of the scotoma conditions, is plotted in Figure 6. The plots for the total viewing time as a function of vertical display position, collapsed across horizontal position, are also shown in Figure 6. Subjects concentrated the majority of processing time on the objects rather than on the space between them, with the majority of time in all conditions concentrated on the center of each object. Again, this centralized distribution of processing time held in the scotoma-centered condition, even though there was no object displayed in the region so long as the eyes remained there. However, unlike Experiment 1, there was a target (the placeholder) at which to direct the eyes in Experiment 2.

There was also a tendency, apparent in Figure 6, for the eyes to fixate the regions between objects for a longer time in the scotoma-centered condition than in the scotoma-offset and scotoma-absent conditions. Total fixation times in the intermediate regions between objects were reliably longer in the scotoma-centered condition $(278 \mathrm{msec})$ than in the scotoma-absent $(157 \mathrm{msec})$ and scotoma-offset $(216 \mathrm{msec})$ conditions $[F(2,22)=12.44$, $\left.M S_{\mathrm{e}}=17,576, p<.001\right]$.

Number of entries. Figure 7 shows the mean number of entries into each region as a function of ordinal region position and scotoma condition, and Table 4 shows the mean number of entries into the four object regions, collapsed over region. Overall, subjects entered each object 

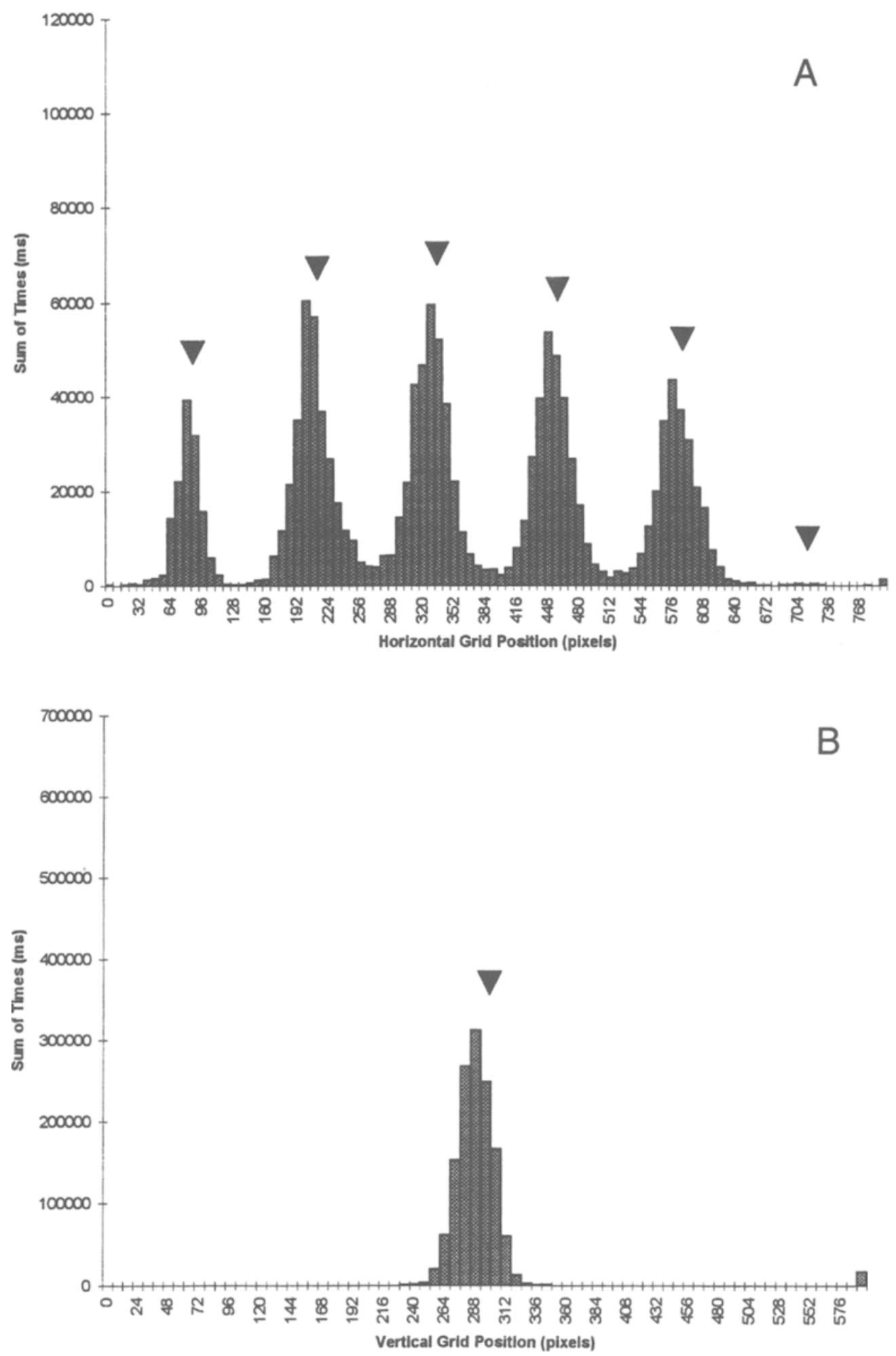

Figure 6. Total viewing time on the arrays as a function of position (in screen pixel coordinates), Experiment 2. Panel A shows horizontal position collapsed over vertical position, and panel B shows vertical position collapsed over horizontal position, for the scotoma-absent condition. Arrows in each panel show the centers of the six regions. (See following pages for equivalent plots for the scotoma-offset condition (panels $C$ and $D$ ] and the scotoma-centered condition [panels $E$ and $F$ ].) 

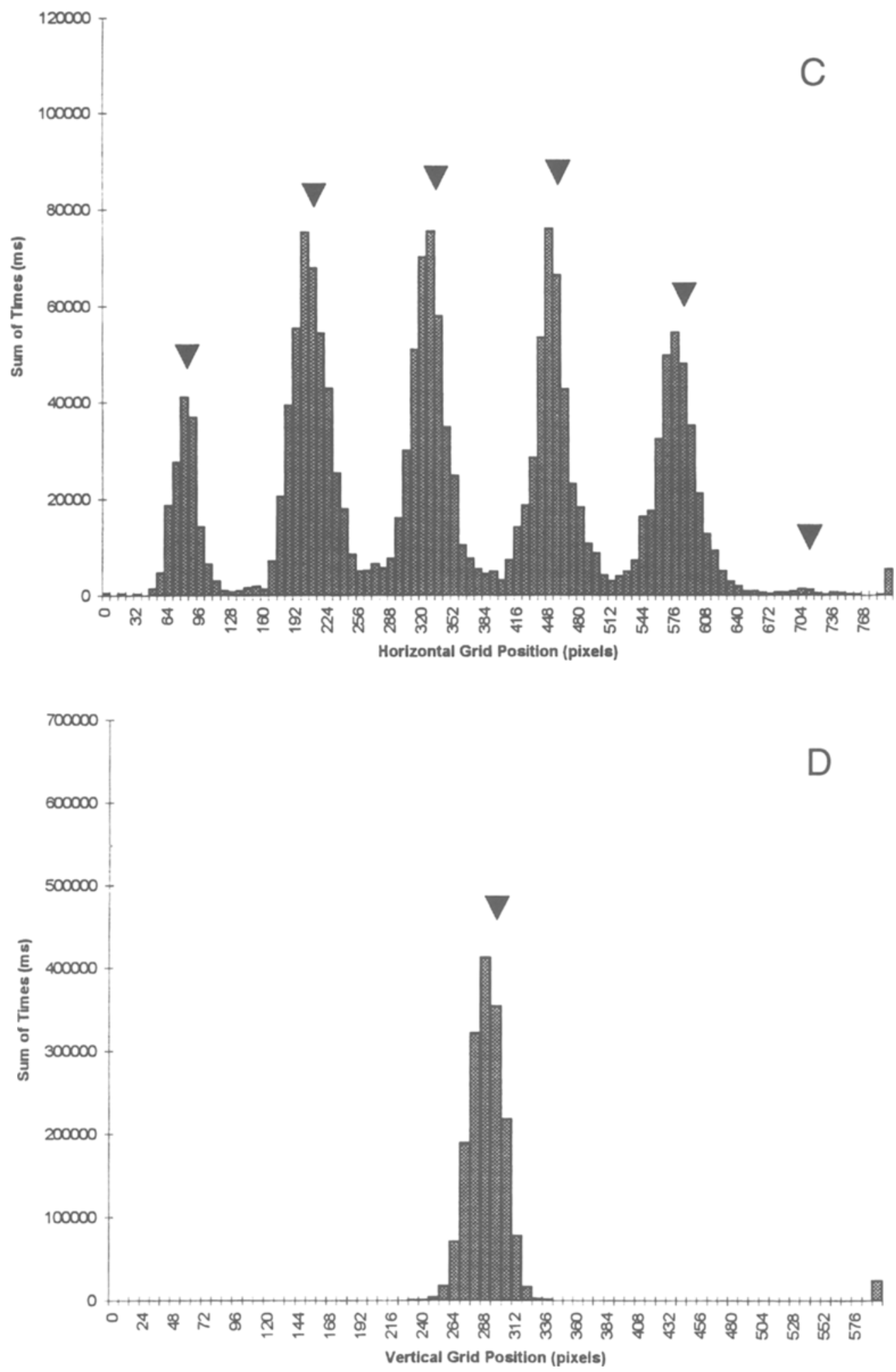

Figure 6 (Continued). Total viewing time on the arrays as a function of position (in screen pixel coordinates), Experiment 2. Panel $C$ shows horizontal position collapsed over vertical position, and panel $D$ shows vertical position collapsed over horizontal position, for the scotoma-offset condition. Arrows in each panel show the centers of the six regions. (See following page for equivalent plots for the scotoma-centered condition (panels $E$ and $F \mid$ and previous page for the scotoma-absent condition (panels $A$ and $B \mid$.) 

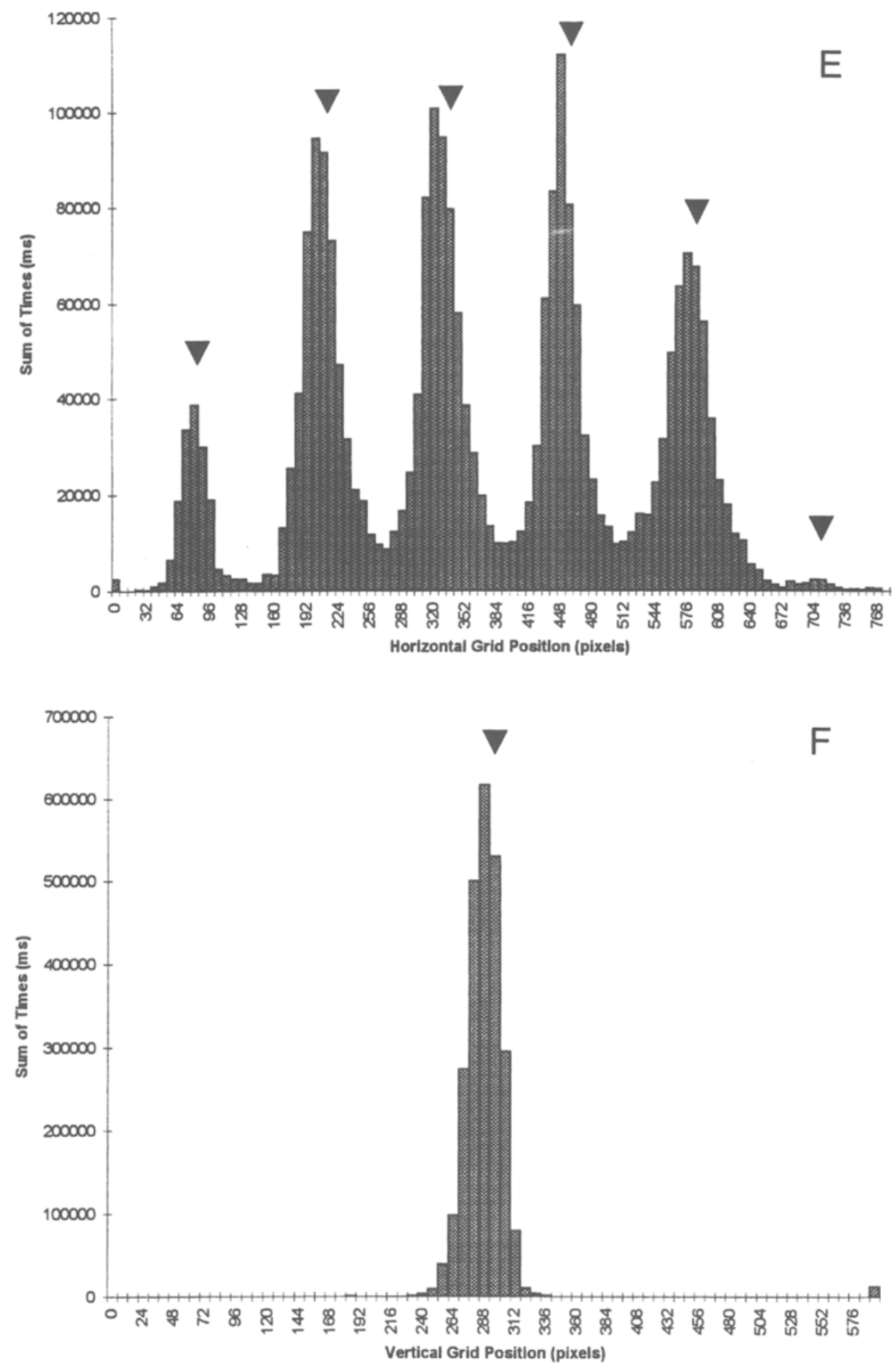

Figure 6 (Continued). Total viewing time on the arrays as a function of position (in screen pixel coordinates), Experiment 2. Panel $E$ shows horizontal position collapsed over vertical position, and panel $F$ shows vertical position collapsed over horizontal position, for the scotoma-centered condition. Arrows in each panel show the centers of the six regions. (See preceding pages for equivalent plots for the scotoma-absent condition [panels $A$ and $B$ ] and the scotoma-offset condition [panels $C$ and $D$ ].) 


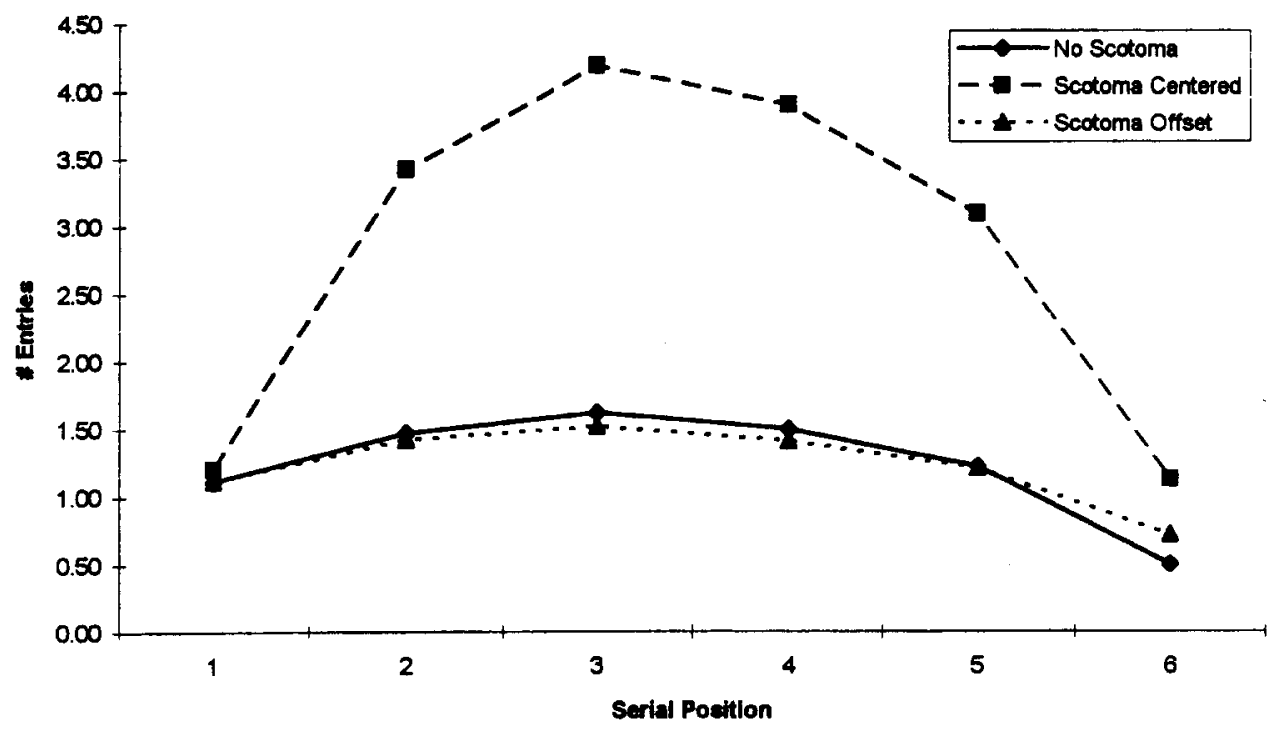

Figure 7. Mean number of entries into the six regions of the arrays as a function of ordinal region position and scotoma condition, Experiment 2.

region 2.16 times. While the number of entries in the scotoma-absent and scotoma-offset conditions was equivalent, there were more entries in the scotoma-centered condition, leading to a main effect of the scotoma condition $\left[F(2,22)=178.9, M S_{\mathrm{e}}=.4422, p<.001\right]$. As found in Experiment 1, this effect was exaggerated for the central two object positions as compared with the first and last object positions, leading to a reliable interaction of scotoma condition and ordinal position $[F(6,66)=11.46$, $\left.M S_{\mathrm{e}}=.0457, p<.001\right]$.

Total fixation time. Figure 8 (top panel) shows the mean total fixation time in each region as a function of ordinal position and scotoma condition; Table 4 shows the mean total fixation time for the four object regions as a function of scotoma condition, collapsed over region. Overall, the mean total fixation time on each object region was $1,093 \mathrm{msec}$. Total fixation time was reliably affected by scotoma condition $\left[F(2,22)=49.87, M S_{\mathrm{e}}=\right.$ $122,818, p<.001]$, with the longest total times in the scotoma-centered condition and somewhat elevated times in the scotoma-offset condition, as compared with the scotoma-absent condition. Simple effects tests based on the pooled error term showed that the mean total fixation time in each scotoma condition was reliably different from the mean total fixation time in each of the other two scotoma conditions $(p s<.05)$. The effect of the scotoma condition was not mediated by ordinal region position $(F<1)$.

Total fixation count. Figure 8 (bottom panel) shows the mean total fixation count for each object as a function of ordinal position and scotoma condition, and Table 4 shows the mean total fixation count for the four object regions as a function of scotoma condition, collapsed over region. Overall, the mean total fixation count on each region was 3.52. These data showed a similar pattern to that of the total fixation time data $[F(2,22)=50.49$, $\left.M S_{\mathrm{e}}=1.3107, p<.001\right]$. This pattern was not mediated by ordinal object position $(F<1)$.

\section{Local Measures of Eye-Movement Patterns}

Gaze duration. Figure 9 (top panel) presents the mean gaze duration in each region as a function of ordinal position and scotoma condition, and Table 4 shows the mean gaze duration on the four object regions as a func-

Table 4

Mean Number of Entries, Total Fixation Time (in Milliseconds), Total Fixation Count, Gaze Duration (in Milliseconds), and Gaze Fixation Count for the Four Line Drawings Collapsed Over Ordinal Position, Experiment 2

\begin{tabular}{lccccc}
\hline $\begin{array}{c}\text { Scotoma } \\
\text { Condition }\end{array}$ & $\begin{array}{c}\text { Number of } \\
\text { Entries }\end{array}$ & $\begin{array}{c}\text { Total } \\
\text { Fixation Time }\end{array}$ & $\begin{array}{c}\text { Fotal } \\
\text { Fixation Count }\end{array}$ & $\begin{array}{c}\text { Gaze } \\
\text { Duration }\end{array}$ & $\begin{array}{c}\text { Gaze } \\
\text { Fixation Count }\end{array}$ \\
\hline Absent & 1.45 & 779 & 2.67 & 599 & 1.99 \\
Offset & 1.39 & 1,020 & 3.04 & 815 & 2.37 \\
Centered & 3.64 & 1,482 & 4.86 & 390 & 1.28 \\
Mean & 2.16 & 1,093 & 3.52 & 601 & 1.88 \\
\hline
\end{tabular}



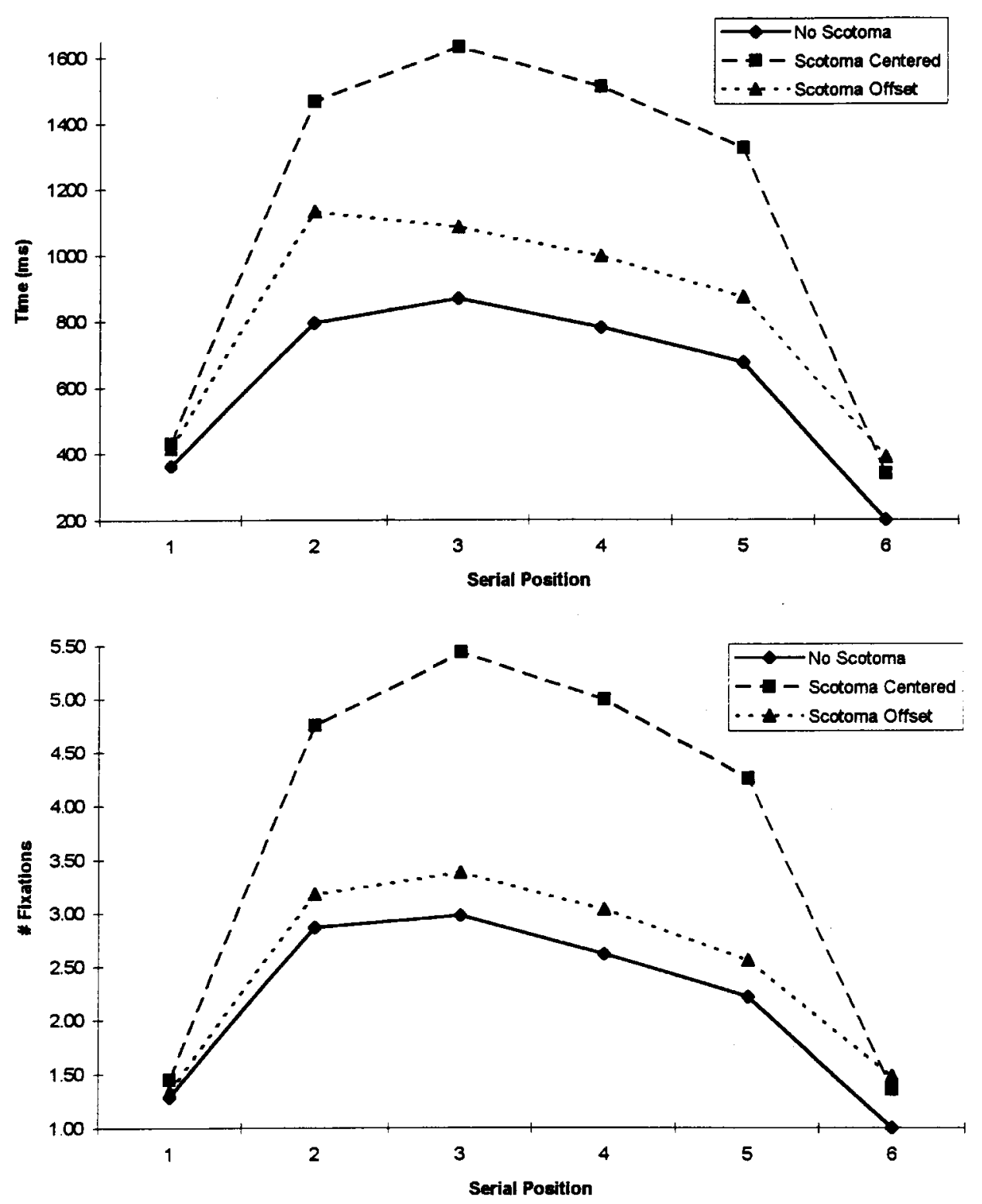

Figure 8. Mean total fixation time in milliseconds (top panel) and mean total fixation count (bottom panel) on the arrays as a function of ordinal region position and scotoma condition, Experiment 2.

tion of scotoma condition, collapsed over region. Mean gaze duration in each object region was $601 \mathrm{msec}$. The scotoma-centered condition produced shorter gaze durations than the scotoma-absent condition, whereas the scotoma-offset condition produced longer gaze durations than the scotoma-absent condition $[F(2,22)=$ $\left.85.53, M S_{\mathrm{e}}=25,276, p<.001\right]$. Simple effects tests based on the pooled error term showed that the mean gaze duration in each scotoma condition was reliably different from the mean gaze duration in each of the other two scotoma conditions ( $p s<.05$ ). Unlike Experiment 1 , this pattern held for all four ordinal object positions, with no interaction between scotoma condition and ordinal object position $\left[F(6,66)=1.580, M S_{\mathrm{e}}=7,096, p>.15\right]$.
Gaze fixation count. Figure 9 (bottom panel) presents the mean gaze fixation count in each region as a function of ordinal region position and scotoma condition, and Table 4 shows the mean gaze fixation count on the four object regions as a function of scotoma condition, collapsed over region. Mean gaze fixation count in each object region was 1.88 . The gaze fixation count data produced a pattern similar to that of the gaze duration data, with the smallest number of fixations in the scotomacentered condition, the largest number in the scotomaoffset condition, and an intermediate number in the scotoma-absent condition $\left[F(2,22)=62.40, M S_{\mathrm{e}}=\right.$ $.2378, p<.001]$. Simple effects tests based on the pooled error term showed that the mean gaze fixation count was 

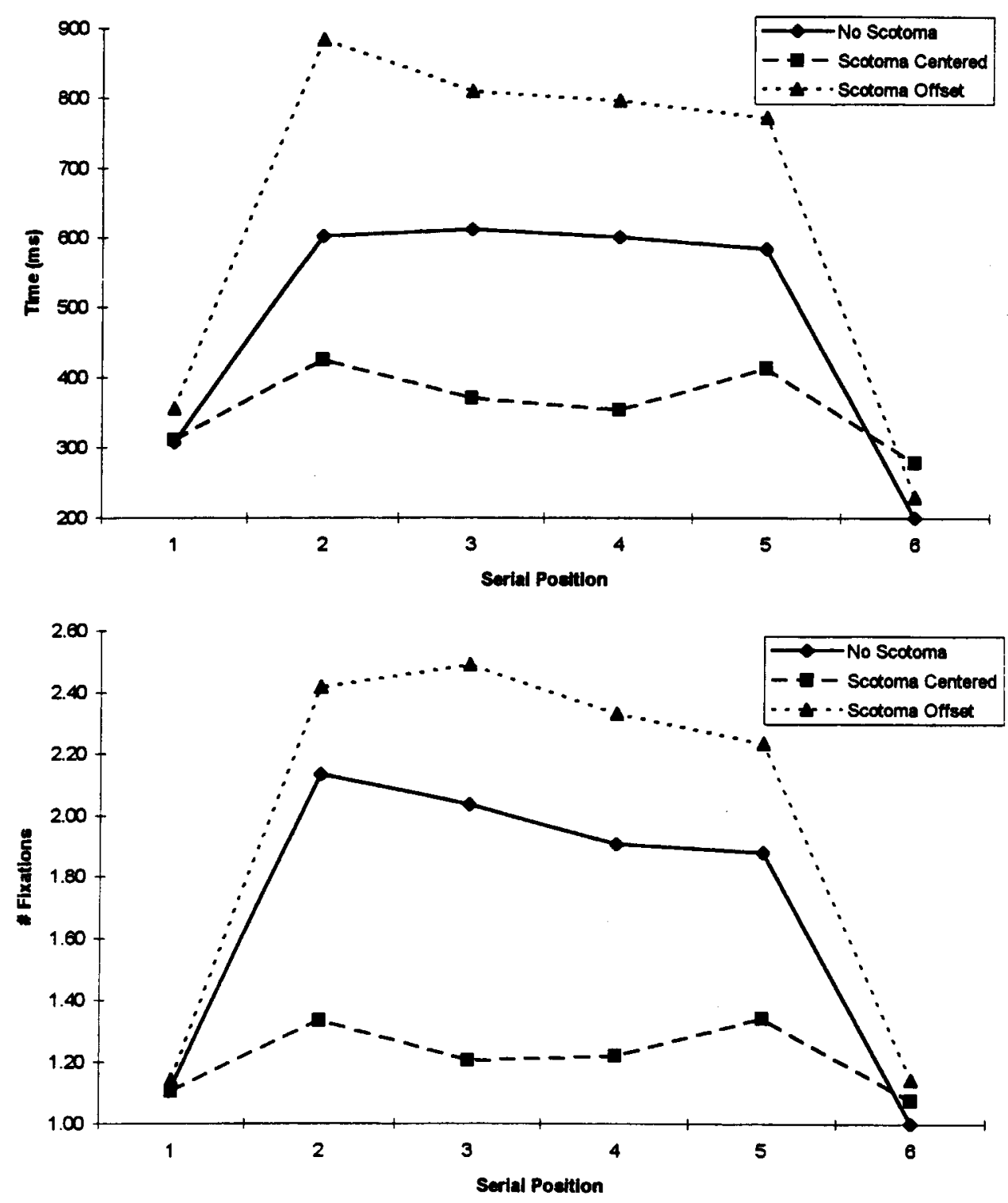

Figure 9. Mean gaze duration in milliseconds (top panel) and mean gaze fixation count (bottom panel) on the arrays as a function of ordinal region position and scotoma condition, Experiment 2.

reliably different from the mean gaze fixation count in each of the other two scotoma conditions $(p s<.05)$. This pattern was not mediated by ordinal object position $\left[F(6,66)=1.850, M S_{\mathrm{e}}=.0529, p>.10\right]$.

\section{Discussion}

Once again, the high test-probe accuracy showed that subjects were able to identify the objects in all of the scotoma conditions, including the scotoma-centered condition, although there was a reliable $10.5 \%$ decrease $(95.1 \%$ to $84.6 \%$ ) for the hit rates and an unreliable $2.1 \%$ increase $(2.1 \%$ to $4.2 \%)$ for the false-alarm rates in the foveal scotoma condition in comparison with the control condition. In addition to this drop in performance, viewing behavior was changed in regular ways when foveal infor- mation was absent, as seen in the eye-movement measures. As in Experiment 1, the global eye-movement measures showed that a central scotoma produced more entries, greater total fixation time, and more individual fixations in each region as compared with the scotomaoffset and scotoma-absent conditions. Also consistent with Experiment 1, eye-movement behavior was affected by the presence of a central scotoma at the earliest moments of object viewing: the local measures showed that the central scotoma led to shorter gaze durations and fewer gaze fixations than did the scotoma-offset and scotoma-absent conditions.

The peripheral scotoma condition also produced viewing behavior that was very similar to that observed in Experiment 1 . In the global measures, the offset scotoma 
produced the same number of entries, but more time and more individual fixations in each region than did the scotoma-absent control condition. This pattern is the same as that observed in Experiment 1. In the local measures, more gaze fixations were made and gaze durations were longer in each region in the scotoma-offset condition than in the control condition. This pattern is similar to that observed in Experiment 1, except that the number of gaze fixations in Experiment 1 was the same in the scotoma-offset and scotoma-absent conditions. Overall, the pattern of data again suggests a much smaller effect of the offset scotoma than of a central scotoma.

A central purpose of Experiment 2 was to determine whether the markedly changed viewing patterns observed in the scotoma-centered condition of Experiment 1 were due to the lack of useful object information at the foveae (the visual encoding hypothesis) or to the lack of any stimulation at all at the foveae (the oculomotor hypothesis). These hypotheses were tested by presenting a placeholder at fixation in the scotoma-centered condition. If the changes in eye-movement patterns were due to the lack of any foveal stimulation in the scotomacentered condition, then those patterns would be expected to disappear with the use of placeholders. In contrast, if those patterns of viewing behavior were due to the lack of useful object information rather than the lack of foveal stimulation, then the same pattern should be observed with the placeholders present. The eye-movement patterns in Experiment 2 were very similar to those of Experiment 1 , supporting the visual encoding hypothesis.

In summary, the data from Experiment 2 suggest that while performance based on object encoding may decline when foveal information is absent, performance can still be quite good as long as compensatory extrafoveal encoding can take place. These data converge with those from Experiment 1 in supporting the hypothesis that foveal processing is beneficial, but not strictly necessary, for object encoding in a free-viewing task.

\section{GENERAL DISCUSSION}

The two experiments reported in this article were designed to investigate the role of foveal analysis in object identification. Three general classes of hypotheses were considered: (1) foveal processing is necessary for object encoding; (2) foveal processing is beneficial, but not necessary, for encoding; and (3) foveal processing is neither necessary nor beneficial for object encoding. To investigate this issue, a display change paradigm was used to produce an artificial scotoma during dynamic viewing of an array of four line drawings of objects. The position of the scotoma was updated in real time as a function of the viewer's current line of regard so that the scotoma moved with the position of the eyes.

Three scotoma conditions were used in the two experiments. In the scotoma-centered condition, objects were visible only peripherally; as soon as the eyes were directed toward an object, it disappeared from the display. In the scotoma-offset condition, the object immediately to the right of the currently foveated object disappeared as the eyes moved through the display. In the scotomaabsent (control) condition, all objects were visible regardless of the line of regard. In Experiment 1, the scotoma was created by replacing the appropriate object with the gray background field; in Experiment 2, the object was replaced with a placeholder. The results in both experiments were consistent with the hypothesis that foveal information is beneficial but not necessary for object encoding: Performance in an object-identification task was equivalent in Experiment 1 and, though reduced, still well above chance in Experiment 2, whether a foveal scotoma was present or not; fixation times and eyemovement patterns, however, were disrupted in both experiments by a foveal scotoma. The nature of this disruption can be summarized by the general statement that subjects moved their eyes around more and looked longer at the displays when foveal information was not available.

The foveal scotoma results offer an interesting contrast to those that have been observed in reading. Rayner and Bertera (1979) showed that readers could report fewer than $10 \%$ of the words in a sentence (and could report none of the words on $18 \%$ of the trials) when an artificial scotoma eliminated the text within the central $2^{\circ}$ of vision. (They also found an increased number of fixations and increased fixation durations with the artificial foveal scotoma, consistent with the results reported here.) The conclusion was that reading is nearly impossible without central vision. The present results indicate that object identification does not suffer a similar fate. The objects in the present study were about the size of a 4- to 5-letter word in the Rayner and Bertera (1979) study (they displayed text at 3 characters per degree) and, in the scotoma-centered condition, the nearest edge of a visible object was about $1.65^{\circ}$ from fixation, equivalent to the scotoma size of about 10 characters in the Rayner and Bertera study. However, accuracy at reporting the words in the 10-character scotoma condition of Rayner and Bertera's study was about $10 \%$, whereas performance at reporting the objects with a scotoma of similar size in the present study ranged from $94 \%$ to $85 \%$ across the two experiments. Thus, under conditions in which word identification is nearly impossible, the identification of line drawings of objects is very good. This difference probably reflects the fact that distinctions between letters in text rely on very high spatial frequency information, whereas objects can be identified on the basis of lower frequency contour patterns.

Another contrast with reading concerns average fixation durations. It is well known that the average fixation duration on words during reading is about $225 \mathrm{msec}$ (Rayner \& Pollatsek, 1989). However, far less is known about the average fixation duration on objects during free viewing. In the present study, we can compute average fixation durations for the first encounter with each object (average duration of each gaze fixation = gaze duration/ gaze fixation count) and for all of the time spent on each object (average duration of each total time fixation $=$ total fixation time/total fixation count). Examining just 
the scotoma-absent control condition, the average duration of each gaze fixation was $328 \mathrm{msec}$ in Experiment 1 and $301 \mathrm{msec}$ in Experiment 2; the average duration of each total time fixation was $326 \mathrm{msec}$ in Experiment 1 and $292 \mathrm{msec}$ in Experiment 2. These averages are thus longer than those typically observed in reading.

The present findings can also be compared with the results of a recent foveal masking study reported by van Diepen, De Graef, and d'Ydewalle (1995). In that study, subjects viewed line drawings of scenes while their eye movements were recorded. In the masking condition, a pattern mask, composed of pixels of random brightness (from black to light gray), was presented at fixation, effectively creating a foveal scotoma. In the control condition, no mask was presented. On a given masking trial, the mask was one of two sizes $\left(1.5^{\circ}\right.$ wide $\times 1.0^{\circ}$ high and $2.5^{\circ}$ wide $\times 1.7^{\circ}$ high), and could be displayed, following the onset of the fixation, by a delay of $15,45,75$, or $120 \mathrm{msec}$. Consistent with the present study, total viewing time in the 15-msec mask delay condition (the condition closest in timing to that used in the experiments reported above) was longer when a mask was present than when it was not. This effect was greater for the larger than for the smaller mask. The masking effect disappeared with mask delays longer than $45 \mathrm{msec}$, presumably because object analysis could be accomplished by using the information acquired from the fovea during the initial 45-50 msec of a fixation (see also Rayner, Inhoff, Morrison, Slowiaczek, \& Bertera, 1981, for a similar effect in reading). ${ }^{3}$

Van Diepen et al. (1995) also found that the distributions of fixation durations were affected by the masking condition: they observed a dip in the distributions about $120 \mathrm{msec}$ following onset of the mask, regardless of the mask onset delay. To determine whether any such changes to the fixation-duration distributions were produced in the present study, all individual fixations on the arrays in each scotoma condition were segregated into $20-\mathrm{msec}$ bins. Frequencies for each bin were then divided by the total number of fixations in that condition. The resulting distributions for each experiment are plotted in Figure 10. As can be seen, no dips in the distributions were observed in the scotoma-centered condition compared with the scotoma-absent condition. As van Diepen et al. suggested, the onset of the foveal mask during a fixation in their study probably captured attention, increasing the durations of fixations that would normally have terminated about $120 \mathrm{msec}$ later.

The distributions shown in Figure 10 were relatively similar across the scotoma conditions, with a greater spread of fixation durations in the scotoma-absent and scotoma-offset conditions than in the scotoma-centered condition, leading to a relatively high and narrow peak in the latter condition. It appears that when a foveal scotoma eliminated useful foveal information in our study, fixation durations showed less variability. This finding is consistent with the hypothesis that fixation-duration variability is partially controlled by foveal object analysis (Henderson, 1993): when a foveal object is present, fixa- tion durations vary with the processing difficulty of that object; when no foveal object is present, there is no variation in foveal difficulty, and so one source of fixation variability is eliminated.

An interesting finding in Experiment 1 was that the number of individual fixations made on an object upon initially landing on that object (gaze fixation count) and the total duration of those initial fixations (gaze duration) was smaller when a foveal scotoma was present than when it was not. We considered two possible explanations for this effect and contrasted them in Experiment 2. According to the visual encoding hypothesis, the eyes moved away from the (empty) object position more quickly in the central-scotoma condition because attention to the extrafoveal objects tended to draw the eyes. According to the oculomotor hypothesis, the eyes moved away because there was no visual pattern stimulation of any kind at the fovea. Consistent with the visual encoding hypothesis but contrary to the oculomotor hypothesis, the same quick movement away from the object position was observed in Experiment 2 when a patterned placeholder was displayed at fixation in the scotoma-centered condition.

In addition to the disruption caused by a foveal scotoma, there was also some cost associated with a scotoma in the periphery. This cost was not observed in the accuracy data, but was observed in eye-movement behavior. Unlike the scotoma-centered condition, this disruption was manifested as a general increase in processing time in the scotoma-offset condition, as compared with the scotoma-absent condition, including an increase in initial gaze duration. The most straightforward explanation for this effect is that fixation times increased in the scotoma-offset condition because less extrafoveal information had been available from each object prior to its fixation. This explanation is consistent with the studies discussed in the introduction in showing that information is acquired from extrafoveal objects prior to their fixation (Henderson, 1992a; Henderson et al., 1987, 1989; Pollatsek et al., 1984; Pollatsek, Rayner, \& Henderson, 1990). It is also consistent with the present results in showing that viewers can identify objects on the basis of extrafoveal information alone. Removal of extrafoveal information in the scotoma-offset condition removed a source of information that is typically used during object identification and consequently increased the amount of time needed to encode the object once it was fixated.

It is interesting that the majority of viewing time in both experiments was spent on or near the centers of the objects rather than on the boundaries of the objects or the space between them. While such a pattern would be expected in the scotoma-absent condition (because the fixated region contained an object), it is noteworthy that a similar pattern was also observed in the scotomacentered condition of Experiment 1, where the fixated region contained only the gray background field. We suspect that there are two causes of this effect. First, viewers were undoubtedly directing their eyes to the centers of the objects when they were visible in the periph- 

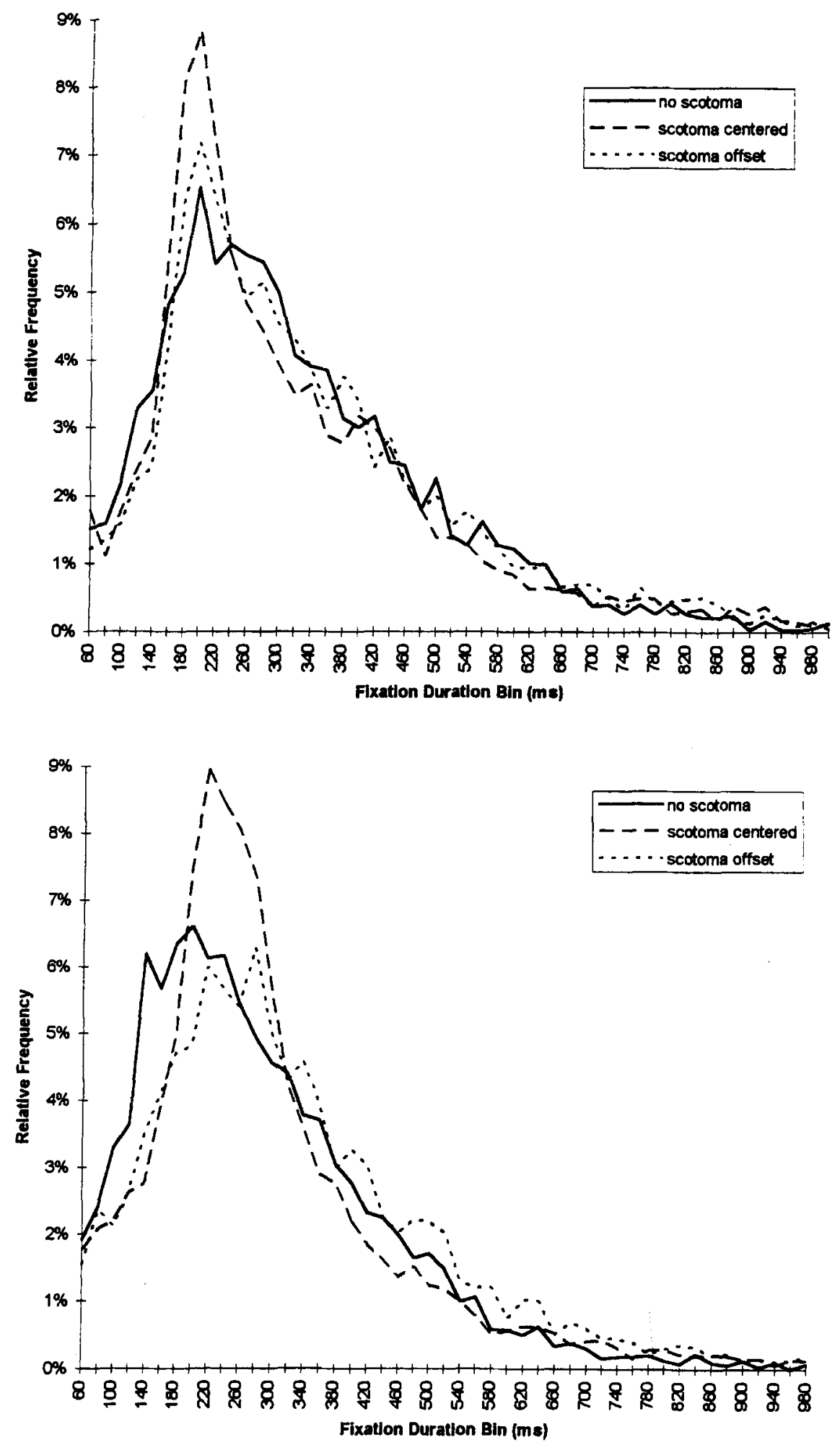

Figure 10. Fixation duration distributions, Experiment 1 (top panel) and Experiment 2 (bottom panel). 
ery, leading to more fixations and so longer time on the centers of those regions. Second, it may be that subjects were spending the majority of time with their eyes centered in the region so that they could pick up compensatory extrafoveal information from the objects in the two flanking regions.

In summary, the present study suggests that foveal information is beneficial but not necessary for object encoding, at least under the display conditions used here (relatively small objects placed relatively close together in the visual field). In both experiments, object identification was very good in the absence of foveal information. At the same time, the eye-movement patterns were disrupted in a manner consistent with compensatory extrafoveal processing. The degree to which additional extrafoveal processing can compensate for the loss of foveal object information would appear to rely on at least two factors. First, compensation can be complete only if the subject engages in sufficient extrafoveal analysis, and this in turn is presumably based on an internal criterion set by the subject. When the criterion is set such that sufficient extrafoveal processing takes place, compensation will be complete and performance will not suffer, as observed in Experiment 1. However, when the criterion is set too low and compensation is incomplete, performance will suffer, as observed in Experiment 2 . Second, the degree to which additional extrafoveal processing can compensate for foveal information loss will depend on the nature of the viewing task. In a task requiring object identification, compensation may be complete. On the other hand, in a task requiring the extraction of fine detail, the amount of compensation that is possible will be limited by extrafoveal visual acuity.

Finally, the finding that identification accuracy was affected relatively little by the presence of a central scotoma while eye-movement patterns showed consistently large effects indicates that eye-movement behavior can provide a unique source of evidence concerning basic perceptual and cognitive processes in object perception.

\section{REFERENCES}

ANTES, J. R. (1974). The time course of picture viewing. Journal of Experimental Psychology, 103, 62-70.

Buswell, G. T. (1935). How people look at pictures. Chicago: University of Chicago Press.

Crane, H. D. (1994). The Purkinje image eyetracker, image stabilization, and related forms of stimulus manipulation. In D. H. Kelley (Ed.), Visual science and engineering: Models and applications (pp. 15-89). New York: Macel Dekker.

Crane, H. D., \& Steele, C. M. (1985). Generation-V dual-Purkinjeimage eyetracker. Applied Optics, 24, 527-537.

De Graef, P., Christiaens, D., \& D'Ydewalle, G. (1990). Perceptual effects of scene context on object recognition. Psychological Research, 52, 317-329.

FisCher, B., \& RAMSPERGER, E. (1984). Human express saccades: Extremely short reaction times of goal directed eye movements. Experimental Brain Research, 57, 191-195.

FisCHER, B., \& WEBER, H. (1993). Express saccades and visual attention. Behavioral \& Brain Sciences, 16, 553-610.

Friedman, A. (1979). Framing pictures: The role of knowledge in automatized encoding and memory for gist. Journal of Experimental Psychology: General, 108, 316-355.
Henderson, J. M. (1992a). Identifying objects across saccades: Effects of extrafoveal preview and flanker object context. Journal of Experimental Psychology: Learning, Memory, \& Cognition, 18, 521530

Henderson, J. M. (1992b). Visual attention and eye movement control during reading and picture viewing. In $\mathrm{K}$. Rayner (Ed.), Eye movements and visual cognition (pp. 260-283). New York: SpringerVerlag.

Henderson, J. M. (1993). Eye movement control during visual object processing: Effects of initial fixation position and semantic constraint. Canadian Journal of Experimental Psychology, 47, 7998.

Henderson, J. M., Dixon, P., Petersen, A., Twilley, L., \& Ferreira, F. (1995). Evidence for the use of phonological representations during transsaccadic word recognition. Journal of Experimental Psychology: Human Perception \& Performance, 21, 82-97.

Henderson, J. M., \& Ferreira, F. (1990). Effects of foveal processing difficulty on the perceptual span in reading: Implications for attention and eye movement control. Journal of Experimental Psychology: Learning, Memory, \& Cognition, 16, 417-429.

Henderson, J. M., Pollatsek, A., \& Rayner, K. (1987). The effects of foveal priming and extrafoveal preview on object identification. Journal of Experimental Psychology: Human Perception \& Performance, 13, 449-463.

Henderson, J. M., Pollatsek, A., \& Rayner, K. (1989). Covert visual attention and extrafoveal information use during object identification. Perception \& Psychophysics, 45, 196-208.

Loftus, G. R., \& MACKworT H, N. H. (1978). Cognitive determinants of fixation location during picture viewing. Journal of Experimental Psychology: Human Perception \& Performance, 4, 565-572.

Mackworth, N. H., \& Morand, A. J. (1967). The gaze selects informative details within pictures. Perception \& Psychophysics, 2 , 547-552.

Nelson, W. W., \& Loftus, G. R. (1980). The functional visual field during picture viewing. Journal of Experimental Psychology: $\mathrm{Hu}$ man Learning \& Memory, 6, 391-399.

O'Regan, J. K. (1992). Optimal viewing position in words and the strategy-tactics theory of eye movements in reading. In $\mathrm{K}$. Rayner (Ed.), Eye movements and visual cognition: Scene perception and reading (pp. 333-354). New York: Springer-Verlag.

PARKER, R. E. (1978). Picture processing during recognition. Journal of Experimental Psychology: Human Perception \& Performance, 4 , 284-293.

Pollatsek, A., Rayner, K., \& Collins, W. E. (1984). Integrating pictorial information across eye movements. Journal of Experimental Psychology: General, 113, 426-442.

Pollatsek, A., Rayner, K., \& Henderson, J. M. (1990). Role of spatial location in integration of pictorial information across saccades. Journal of Experimental Psychology: Human Perception \& Performance, 16, 199-210.

RAYNER, K. (1975). The perceptual span and peripheral cues in reading. Cognitive Psychology, 7, 65-81.

RAYNER, K., \& Bertera, J. H. (1979). Reading without a fovea. Science, 206, 468-469.

Rayner, K., Inhoff, A. W., Morrison, R. E., SlowiaczeK, M. L., \& BERTERA, J. H. (1981). Masking of foveal and parafoveal vision during eye fixations in reading. Journal of Experimental Psychology: Human Perception \& Performance, 7, 167-179.

RAYNER, K., MCConkie, G. W., \& Ehrlich, S. (1978). Eye movements and integrating information across fixations. Journal of Experimental Psychology: Human Perception \& Performance, 4, 529-544.

RAYNER, K., \& PollatSEK, A. (1989). The psychology of reading. Englewood Cliffs, NJ: Prentice-Hall.

SNODGRass, J. G., \& VANDERWART, M. (1980). A standardized set of 260 pictures: Norms for name agreement, image agreement, familiarity, and visual complexity. Journal of Experimental Psychology: Human Learning \& Memory, 6, 174-215.

Van Diepen, P. M. J., De Graef, P., \& D'Ydewalle, G. (1995). Chronometry of foveal information extraction during seene perception. In J. M. Findlay, R. Walker, \& R. W. Kentridge (Eds.), Eye movement research: Mechanisms, processes, and applications (pp. 349362). Amsterdam: Elsevier. 
VITU, F., O'Regan, J. K., INHOFF, A. W., \& TopolSKI, R. (1995). Mindless reading: Eye-movement characteristics are similar in scanning strings and reading texts. Perception \& Psychophysics, 57, 352-364. YARBUS, A. L. (1967). Eye movements and vision. New York: Plenum.

\section{NOTES}

1. There were two reasons why the region to the right of the currently fixated region was deleted (rather than some other region) in the scotoma-offset condition. First, only a single region, rather than the two regions flanking the fixated region, was deleted so that the total amount of the image that changed would be equated in the scotomacentered and scotoma-offset conditions. Second, the region to the right, rather than to the left, was deleted in order to equate as closely as possible the degree to which regions likely to be attended would be changed in the scotoma-centered and scotoma-offset conditions. Under the assumptions that viewers would tend to move from left to right through the arrays and that they would attend to the region currently at fixation and the region about to be fixated next (Henderson, 1992b; Henderson \& Ferreira, 1990; Henderson et al., 1989), the regions most likely to be attended during a trial would be the currently fixated region and the region to the right of that region.
2. Another commonly used local measure of processing difficulty during object encoding is first-fixation duration (De Graef et al., 1990; Henderson et al., 1989). There is currently some dispute in the reading literature concerning the degree to which first-fixation duration reflects low-level oculomotor factors (O'Regan, 1992; Vitu, O'Regan, Inhoff, \& Topolski, 1995), high-level cognitive factors (Henderson \& Ferreira, 1990; Henderson et al., 1987; Rayner \& Pollatsek, 1989), or both. Evidence from the present set of experiments bearing on this controversy will be presented in a separate report.

3. There were a number of differences between the van Diepen et al. (1995) and the present study, so comparisons must be made with caution. For example, van Diepen et al. presented a pattern mask at the fovea, while we presented either nothing (the background field) or a simple placeholder, and while their display changes took place following each saccade and were not accomplished until a fixation had begun, our display changes took place only during boundary crossings and so were accomplished during saccades. Also, van Diepen et al. did not report local measures of eye-movement behavior such as gaze duration or gaze fixation count.

(Manuscript received October 18, 1995; revision accepted for publication April 6, 1996.) 